ARTICLE

\title{
Cisplatin-mediated activation of glucocorticoid receptor induces platinum resistance via MAST1
}

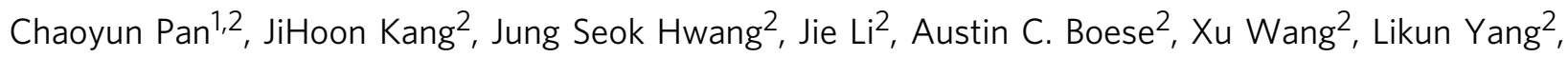
Titus J. Boggon (10 ${ }^{3}$, Georgia Z. Chen², Nabil F. Saba², Dong M. Shin², Kelly R. Magliocca ${ }^{4}$, Lingtao $\operatorname{Jin}^{5}$ \& Sumin Kang (10) 2 压

Agonists of glucocorticoid receptor (GR) are frequently given to cancer patients with platinum-containing chemotherapy to reduce inflammation, but how GR influences tumor growth in response to platinum-based chemotherapy such as cisplatin through inflammationindependent signaling remains largely unclear. Combined genomics and transcription factor profiling reveal that MAST1, a critical platinum resistance factor that reprograms the MAPK pathway, is upregulated upon cisplatin exposure through activated transcription factor GR. Mechanistically, cisplatin binds to C622 in GR and recruits GR to the nucleus for its activation, which induces MAST1 expression and consequently reactivates MEK signaling. GR nuclear translocation and MAST1 upregulation coordinately occur in patient tumors collected after platinum treatment, and align with patient treatment resistance. Co-treatment with dexamethasone and cisplatin restores cisplatin-resistant tumor growth, whereas addition of the MAST1 inhibitor lestaurtinib abrogates tumor growth while preserving the inhibitory effect of dexamethasone on inflammation in vivo. These findings not only provide insights into the underlying mechanism of GR in cisplatin resistance but also offer an effective alternative therapeutic strategy to improve the clinical outcome of patients receiving platinum-based chemotherapy with GR agonists.

\footnotetext{
${ }^{1}$ Department of Biochemistry and Molecular Biology, Zhongshan School of Medicine, Sun Yat-sen University, Guangzhou, China. ${ }^{2}$ Department of Hematology and Medical Oncology, Winship Cancer Institute of Emory, Emory University School of Medicine, Atlanta, GA, USA. ${ }^{3}$ Department of Pharmacology, Yale University School of Medicine, New Haven, CT, USA. ${ }^{4}$ Department of Pathology \& Laboratory Medicine, Emory University School of Medicine, Atlanta, GA, USA. ${ }^{5}$ Department of Anatomy and Cell Biology, University of Florida, College of Medicine, Gainesville, FL, USA.

凶email: smkang@emory.edu
} 
P latinum-based compounds, such as cisplatin or carboplatin, have been the most active clinical drug class for the treatment of a variety of solid tumors including cancers of the ovary, lung, and head and neck for decades ${ }^{1-3}$. However, significant challenges remain with regard to their activity as it is often accompanied by therapy resistance and side effects. Glucocorticoids are first-line antiemetics that are administered during platinum-based chemotherapy regimens ${ }^{4,5}$. The main actions of glucocorticoids occur through the activation of glucocorticoid receptor (GR), also known as nuclear receptor subfamily 3 group C member 1 (NR3C1), which is a ligand-dependent transcription factor belonging to the superfamily of nuclear receptors ${ }^{6}$. GR is typically found in the cytoplasm as a GR-hsp90 heterocomplex, which is assembled by a multiprotein chaperone machinery hsp90/Hop/hsp70/hsp40 ${ }^{7-9}$. The steroidal ligand occupying GR in the ligand-binding domain (LBD) recruits GR to the nucleus ${ }^{8,10}$. Multiple factors are involved in nucleocytoplasmic shuttling of the GR. Abelson helper integration site 1 (Ahil) and tetratricopeptide-repeat (TPR) domain proteins are known to hinder GR nuclear accumulation, while SIRT2 accelerates nuclear translocation of GR by altering hsp90 acetylation that mediates dissociation of hsp90 from the GR complex ${ }^{11-13}$. The nuclear transported homodimer form of GR binds to glucocorticoid response elements (GRE) in the promoters of glucocorticoid responsive genes to activate their transcription ${ }^{14}$. GR induces or represses the transcription of target genes and a series of these target genes are involved in inflammation and immune response ${ }^{15-17}$. Therefore, synthetic derivatives of glucocorticoid such as dexamethasone are widely used for their antiinflammatory and immune-suppressive properties to relieve symptoms, including hypersensitivity, allergic reactions, edema, nausea, vomiting, and other discomforts that may arise during platinum-based treatment ${ }^{15,18,19}$.

Despite the wide usage of GR agonists for co-medication in cancer chemotherapy as supportive care, a precise role of GR activation in response to platinum-based therapy is largely unknown. Growing evidence suggests that dexamethasone may reduce anti-tumor activity of chemotherapeutic agents including paclitaxel or cisplatin. Previous studies demonstrated that dexamethasone treatment results in blunting cellular senescence, which may be linked with inhibition of NF- $\kappa B$ activity and p53 signaling in cisplatin-treated non-small cell lung carcinoma ${ }^{20}$. Moreover, treatment with a GR agonist enhanced tumor growth and resulted in altered gene expression of anti- and pro-apoptotic factors in a paclitaxel-treated xenograft mouse model of breast cancer ${ }^{21}$. Dexamethasone also attenuated antitumor activity of cisplatin and increased expression of adhesion molecules including integrin $\beta 1$ in ovarian cancer cell lines ${ }^{22}$. Conversely, glucocorticoids had minimal effect on cytotoxicity of cisplatin or paclitaxel in a head and neck cancer cell line UMSCC-14C, or even enhanced cytotoxicity of cisplatin by suppressing NF- $\kappa \mathrm{B}$ activation in the cervical cancer cell line $\mathrm{SiHa}$ in vitro 23,24 . Two independent groups have recently demonstrated that the pro-tumorigenic activities including paclitaxel resistance of GR are mediated, at least in part, by the activation of the hippo pathway transducers YAP and TEAD4 in breast cancer $^{25,26}$. However, studies lacked either in vivo validations or clinical correlation assessments, and the detailed mechanisms by which the activation of GR is specifically linked to platinumbased therapy resistance still remain largely elusive.

A group of signaling effectors have been identified as predictive markers of cisplatin resistance. ATPase7A/7B/11B and ERCC1 are involved in pre-target or on-target cisplatin resistance mechanisms, respectively ${ }^{27-32}$. We identified a microtubuleassociated serine/threonine kinase 1 (MAST1) as a common essential driver for cisplatin resistance that provides a post-target cisplatin resistance mechanism through managing the proapoptotic pathway in human cancers, including lung, ovarian, and head and neck cancers ${ }^{33}$. Mechanistically, MAST1 provides cisplatin resistance by replacing the traditional kinase cRaf when cisplatin disrupts the interaction of MEK and cRaf, which reactivates the MAPK pathway and consequently controls the level of pro-apoptotic factor $\mathrm{BIM}^{33}$. Hsp90B was found to stabilize MAST1 by hindering CHIP-mediated ubiquitination at lysine 317 and 545 and preventing proteasomal degradation ${ }^{34}$. In addition, lestaurtinib was identified as a promising MAST1 inhibitor that effectively sensitizes tumor cells to cisplatin in vitro and in vivo in patient-derived xenograft mouse models. However, whether and how MAST1 is induced during treatment for acquired platinum resistance has never been explored.

In this study, through multidisciplinary approaches, we identify a unique GR activation mechanism that is mediated by cisplatin and demonstrate how this activation contributes to cisplatin-resistant tumor growth through MAST1 induction. We also offer a promising therapeutic strategy that may provide an effective regimen for patients who need GR agonists as antiinflammatory medication along with platinum-based therapy.

\section{Results}

Platinum treatment induces gene expression of MAST1. To glean insight into the genetic variation involved in acquired cisplatin resistance, we established an in vivo model of cisplatinresistant cancer by administering a serial dose of cisplatin to mice bearing KB-3-1 human carcinoma cells (Fig. 1a). Through a whole-transcriptome analysis, we identified a large spectrum of genes that are altered greater than 1.2-fold in tumors that acquired cisplatin resistance compared to treatment-naïve tumors (Fig. 1b). The panel included 61 kinase genes, many of which are important for control of chemotherapy resistant cell growth including previously identified PDK2 and CDK2035,36. We identified microtubule-associated serine/threonine kinase 1 (MAST1), as one of the critical cisplatin-induced factors (Fig. 1c). MAST1 confers cisplatin resistance by replacing cRaf to reactivate the MAPK pathway ${ }^{33}$. Induction of the MAST1 gene in xenografted tumors after cisplatin administration in mice was further validated by quantitative RT-PCR and immunoblotting (Fig. 1d). In addition, the expression level of MAST1 was increased upon cisplatin treatment in cancer cell lines including cervical cancer KB-3-1 and ovarian cancer A2780 (Fig. 1e). Consistent with observations in the preclinical setting, comparison of MAST1 expression between paired pre- and post-platinum therapy tumor samples from head and neck squamous cell carcinoma (HNSCC) patients who did not respond to platinum-based chemotherapy further demonstrated that MAST1 expression is induced during treatment (Fig. 1f, g). Non-responders refer to patients with tumor recurrence within 2 years of cisplatin or carboplatin-based chemotherapy. These data indicate that platinum-based drugs such as cisplatin induce gene expression of MAST1 in human cancers.

GR is a transcription factor that stimulates MAST1 expression upon cisplatin exposure. To glean a comprehensive mechanistic insight into how MAST1 is upregulated upon cisplatin exposure, we performed transcription factor (TF) activation profiling using cisplatin-treated or non-treated KB-3-1 cells. The TF Activation Profiling Plate Arrays monitor activities of 96 cellular TFs, including HIF1, p53, and NF- $\kappa$ B, that are known to be essential in regulating cellular gene expression. Among the 96 TFs, six were activated more than 1.8 -fold when cells were treated with cisplatin, including GR/progesterone receptor (PR), NFAT, ATF2, CAR, and CBF (Fig. 2a). To investigate whether any of these TFs 
a

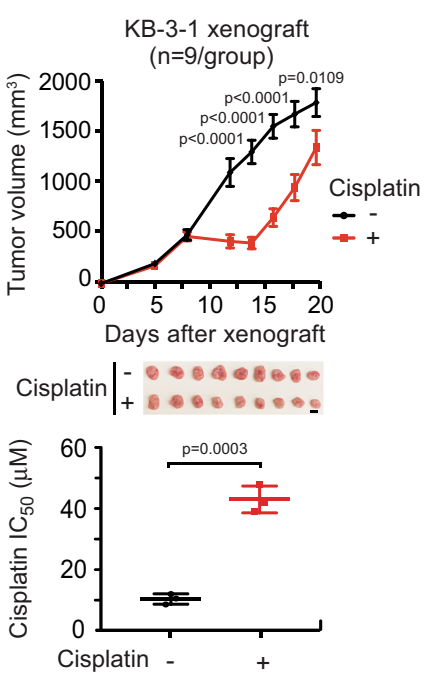

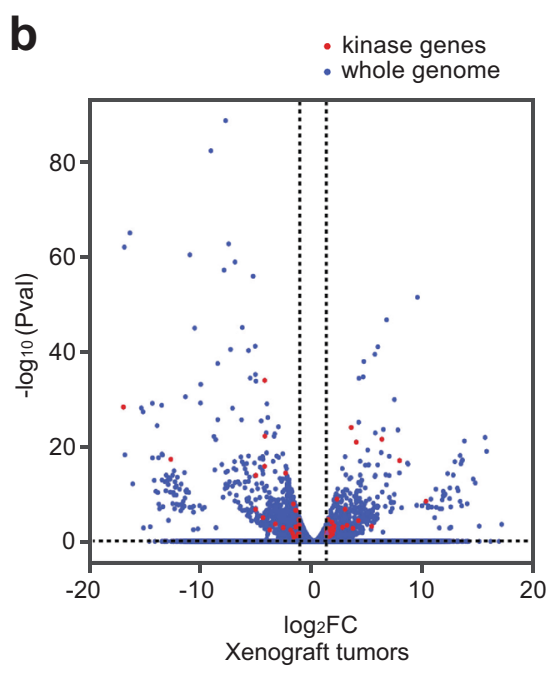

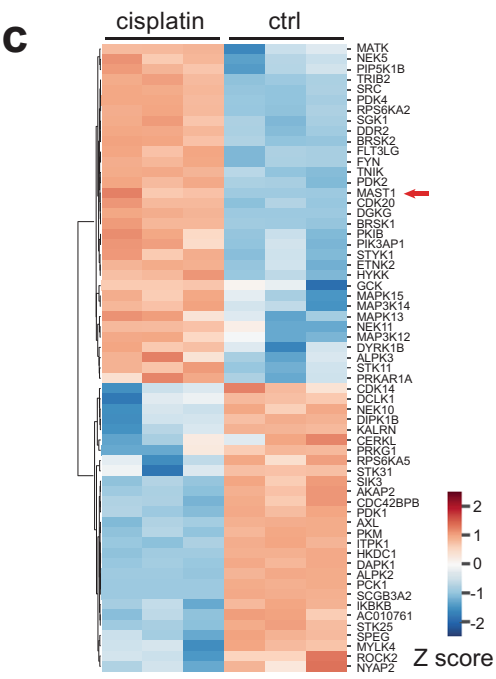

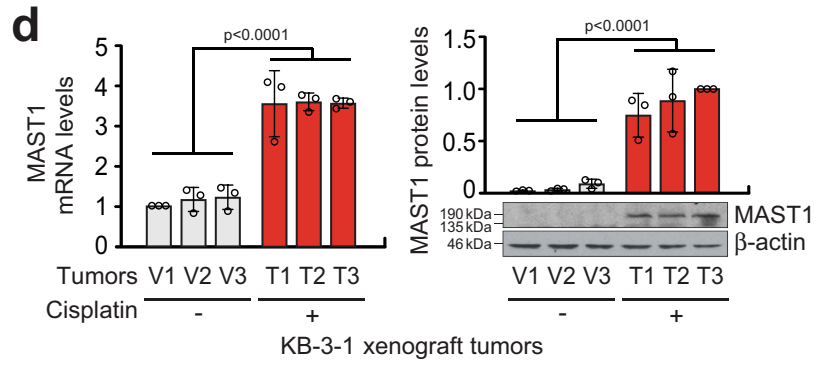

e
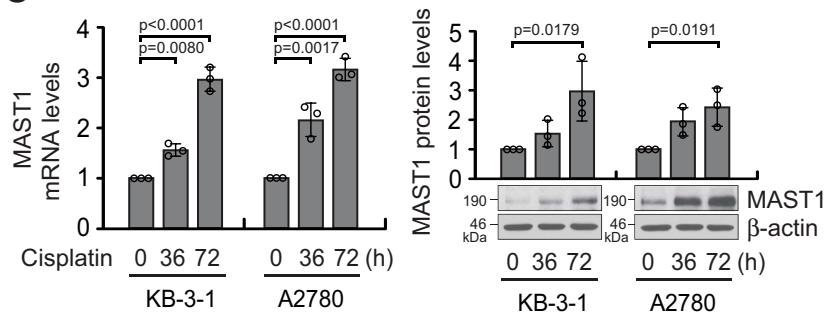

$\mathbf{f}$

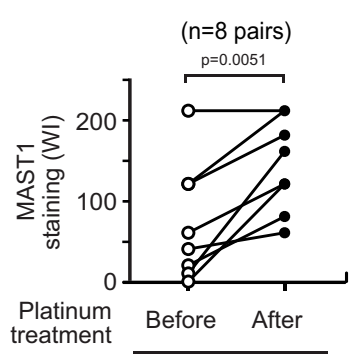

Paired HNSCC patient tumors from non-responders g

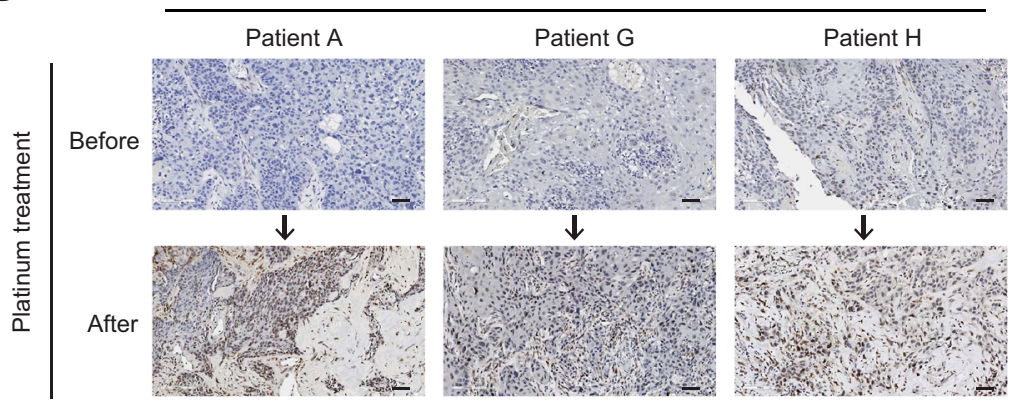

Fig. 1 Cisplatin treatment induces MAST1 in preclinical and clinical patient tumors. a Development of cisplatin-resistant model in vivo. KB-3-1 xenograft mice were treated with 2 doses of cisplatin $(0.5$ and $5 \mathrm{mg} / \mathrm{kg} / \mathrm{i} . \mathrm{p}$. on day 5 and 10). Tumor volumes (top) and cisplatin resistance of three representative xenograft tumors was determined by cisplatin $\mathrm{IC}_{50}$ at the experimental endpoint (bottom). $\mathbf{b}$ Transcriptomic analysis of xenograft tumors collected from mice treated with PBS or cisplatin. RNA-sequencing data are presented as a volcano plot and kinase genes are highlighted in red. $\mathbf{c}$ A heatmap and hierarchical clustering analysis summarizing expression profile of kinase genes with a fold change greater than 1.2. MAST1 is highlighted with an arrow. $\mathbf{d}$ Quantitative RT-PCR and western blot results show increased MAST1 expression in KB-3-1 xenograft mice tumors collected from the cisplatin-treated group (T1-T3) compared to the vehicle-treated group (V1-V3). Three representative tumors from each group were randomly selected for analyses. e The mRNA and protein levels of MAST1 in KB-3-1 and A2780 cells treated with cisplatin $(0.5 \mu \mathrm{g} / \mathrm{ml})$ for the indicated times were determined as d. $\mathbf{f} I \mathrm{HC}$ staining scores of MAST1 in paired primary head and neck squamous cell carcinoma (HSNCC) patient tumors obtained before and after platinum therapy. Patients were considered non-responders to platinum treatment when disease recurred within 2 years after chemotherapy. Weighted index $(\mathrm{WI})=$ positive staining (\%) $\mathrm{x}$ intensity score $(0-3+)$. $\mathbf{g}$ Representative images of $\mathbf{f}$ are shown. Scale bars represent $10 \mathrm{~mm}$ for $\mathbf{a}$ and $50 \mu \mathrm{m}$ for $\mathbf{g}$. Data are mean \pm SEM for tumor volume in a upper panel ( $n=9$ mice/group) and mean \pm SD for cisplatin IC $C_{50}$ in a lower panel ( $n=3$ randomly selected tumors/group). For $\mathbf{d}$ and $\mathbf{e}$, data are mean \pm SD from three independent biological experiments. Statistical analyses were performed by two-way ANOVA for tumor volume and unpaired two-tailed $t$-test for $I C_{50}$ in $\mathbf{a}$ and $\mathbf{d}$, one-way ANOVA for $\mathbf{e}$, and paired two-tailed $t$-test for $\mathbf{f}$. Source data are provided as a Source Data file.

activate the MAST1 promoter, we reduced the expression of the 6 potential candidates by shRNA in KB-3-1 cells and performed a MAST1 promoter reporter assay in the presence of cisplatin. Knockdown of GR but no other TFs attenuated MAST1 promoter activity (Fig. 2b). In agreement, overexpression of GR but not PR, which was undetectable in KB-3-1 cells, enhanced MAST1 gene expression and cell viability upon cisplatin treatment (Fig. 2c). Moreover, chromatin immunoprecipitation assay showed that the MAST1 promoter interacts with GR but not PR in KB-3-1 cells and the binding between MAST1 promoter and GR increases 
a

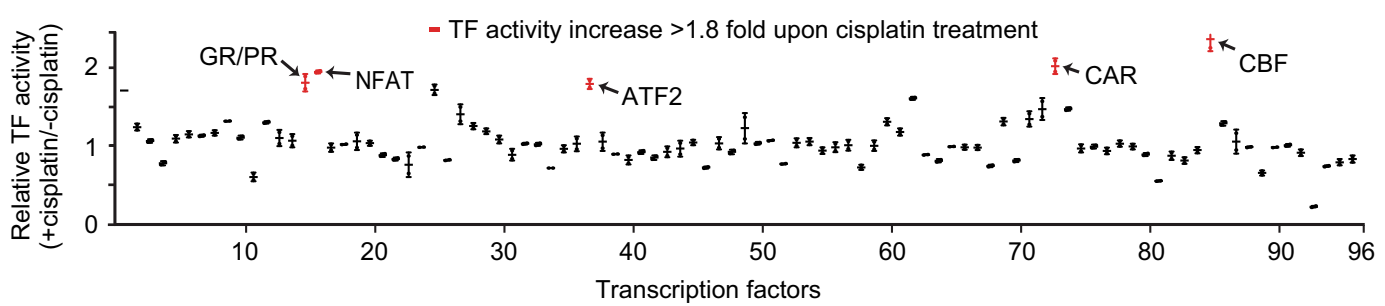

b

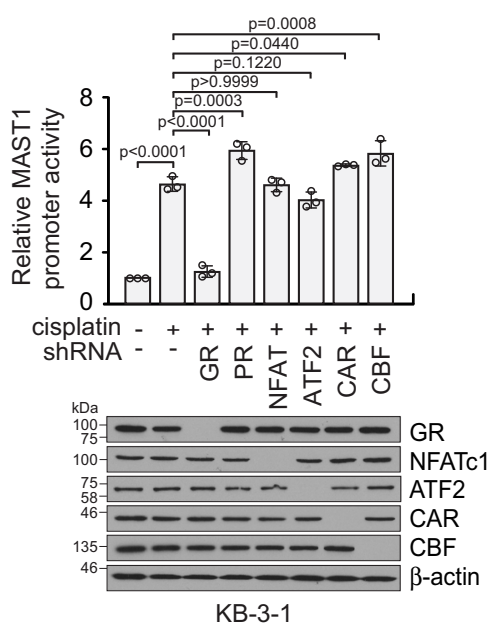

C

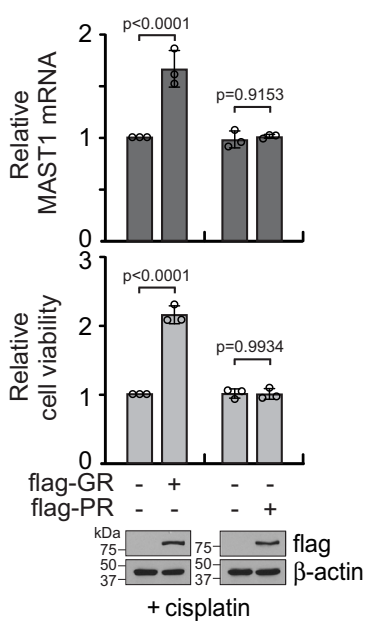

d

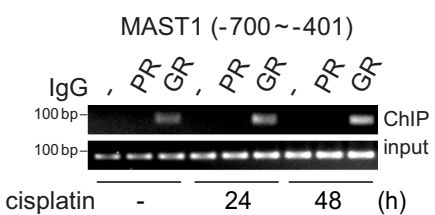

e

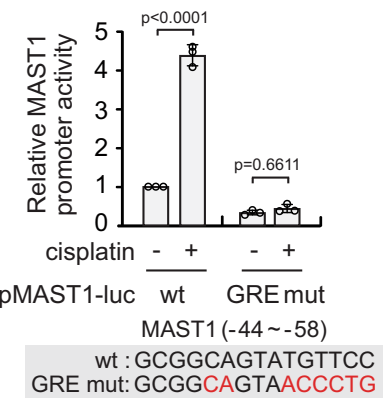

f

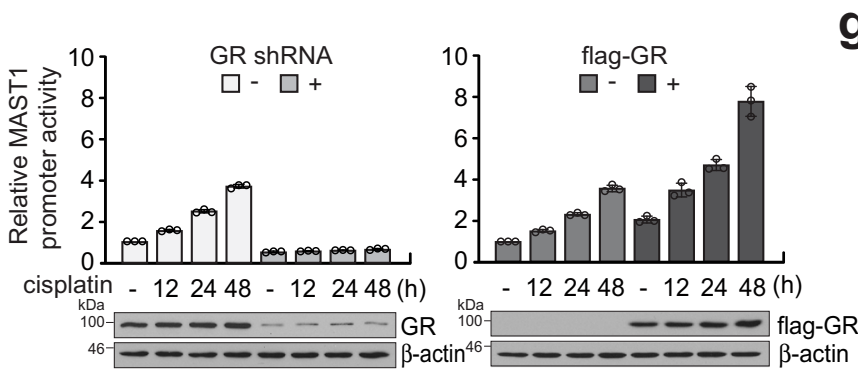

g

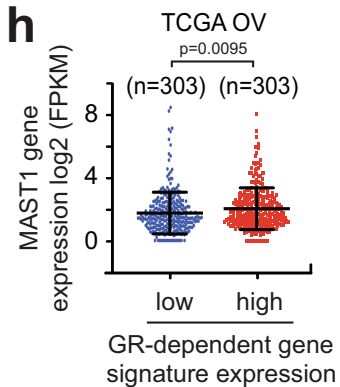

i

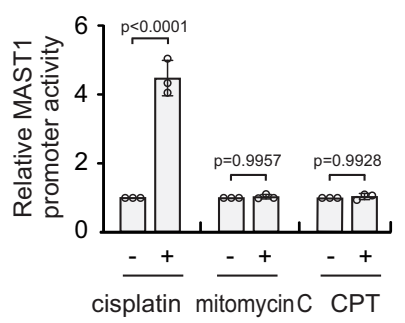

j

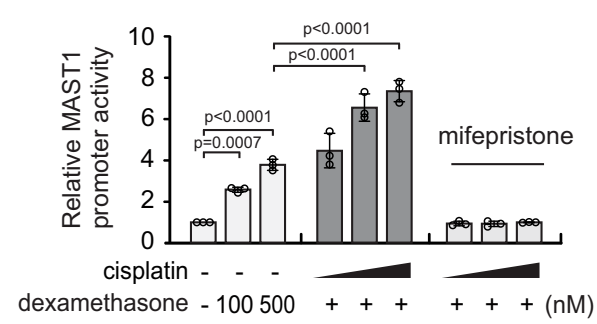

k

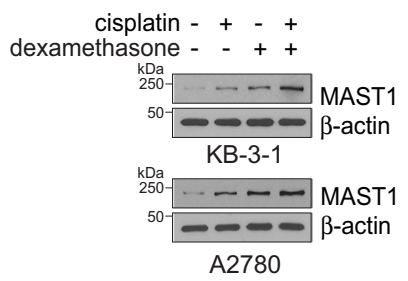

upon treatment with cisplatin (Fig. 2d). GR binds to the glucocorticoid response element (GRE) on DNA within the promoter region and regulates genes ${ }^{37}$. We identified a GRE within the MAST1 promoter sequence at -44 to -58 . To examine whether GR binds to the GRE in the MAST1 promoter region and enhances MAST1 transcription, we generated a binding-deficient mutant form of GRE in the MAST1 promoter sequence by mutating 6 base pairs of the MAST1 promoter reporter construct, pMAST1-luc. Cisplatin induced WT MAST1 promoter activity. However, when the MAST1 promoter carried the GR bindingdeficient mutation in GRE, MAST1 promoter activity was abolished in cancer cells (Fig. 2e). The total amount of MAST1
mRNA increased by cisplatin treatment regardless of promoter activity measured using pMAST1-luc variants (Supplementary Fig. 1a). In addition, modulation of GR affected MAST1 promoter activity in the presence of cisplatin as target downregulation or overexpression of GR, respectively, abolished or enhanced MAST1 promoter activity and mRNA levels in a timedependent manner (Fig. 2f; Supplementary Fig. 1b). In line with the changes seen in MAST1 mRNA, MAST1 protein level correlated with GR level in cisplatin-treated cancer cells (Fig. 2g; Supplementary Fig. 1c). Collectively, these data indicate that cisplatin results in activation of GR and this activated GR binds to the GRE in the MAST1 promoter region to enhance transcription 
Fig. 2 Glucocorticoid receptor (GR), induced by cisplatin, is a transcription factor of MAST1. a Transcriptional factor (TF) activation profiling identifies TFs whose activity is enhanced in KB-3-1 cells in response to cisplatin treatment. b MAST1 promoter activity in KB-3-1 lacking factors whose activity increased more than 1.8-fold by cisplatin. $\mathbf{c}$ Effect of GR or PR overexpression on MAST1 induction and cell viability in KB-3-1 cells treated with cisplatin. $\mathbf{d}$ ChIP assay of GR or PR binding to MAST1 promoter in KB-3-1 cells treated with cisplatin for the indicated times. e Comparison of MAST1 promoter activity in cells expressing WT or glucocorticoid response element (GRE)-mutated MAST1 promoter reporter. KB-3-1 cells were transfected with WT or GRE mutant MAST1 promoter reporter and treated with cisplatin. $\mathbf{f}$ Effect of GR knockdown or overexpression on MAST1 promoter activity in response to cisplatin treatment in KB-3-1 cells. $\mathbf{g}$ Effect of GR knockdown on MAST1 expression in KB-3-1 and A2780 cells in the presence or absence of cisplatin. $\mathbf{h}$ Comparison of MAST1 gene expression in TCGA OV patients stratified by GR-dependent gene signature expression monitoring gene expression of 47 glucocorticoid regulated genes. i Effect of chemotherapy agents on MAST1 promoter activity. KB-3-1 cells carrying MAST1 promoter were treated with sublethal doses of drugs $(1 \mu \mathrm{g} / \mathrm{ml}$ cisplatin, $1 \mu \mathrm{M}$ mitomycin C or camptothecin). $\mathbf{j}$ and $\mathbf{k}$ Induction of MAST1 by cisplatin and dexamethasone. KB-3-1 cells were treated with cisplatin $(0.1,0.2,0.5 \mu \mathrm{g} / \mathrm{ml})$, dexamethasone $(100,500 \mathrm{nM})$, or mifepristone $(10 \mu \mathrm{M})$ for MAST1 promoter activity assay $(\mathbf{j})$. GR antagonist mifepristone was used as a control. KB-3-1 and A2780 cells were treated with cisplatin ( $1 \mu \mathrm{g} / \mathrm{ml})$, dexamethasone ( $500 \mathrm{nM})$, or combination for MAST1 protein induction (k). Data are mean \pm SD from two technical replicates for $\mathbf{a}$, three independent biological experiments for $\mathbf{b}-\mathbf{g}$ and $\mathbf{i}-\mathbf{k}$. Statistical analyses were performed by two-tailed $t$-test for $\mathbf{h}$ and one-way ANOVA for the rest. Source data are provided as a Source Data file.

of MAST1 in cancer cells. Our finding was further validated by analyzing a clinical database. MAST1 levels were higher in ovarian cancer patients with high GR activity compared to patients with low GR activity, which was determined by transcriptome signature of glucocorticoid regulated genes (Fig. 2h). Activation of MAST1 promoter was only observed in cancer cells treated with cisplatin, but not with other DNA damaging agents such as mitomycin $\mathrm{C}$ or camptothecin (CPT), demonstrating that the MAST1 induction occurs specifically through cisplatin (Fig. 2i; Supplementary Fig. 1d). Moreover, cisplatin activated the MAST1 promoter, whereas, treatment with the GR agonist dexamethasone additively further enhanced the promoter activity and MAST1 expression (Fig. 2j, k; Supplementary Fig. 1e, f). Moreover, GR agonists including betamethasone, prednisolone, and triamcinolone showed similar induction of MAST1 as dexamethasone (Supplementary Fig. 2a, b). These data suggest that while cisplatin and GR agonists both activate GR, the activation mechanism may differ.

Cisplatin binds to and nuclear transports GR. To explore the molecular mechanism by which the transcription factor activity of GR is enhanced by cisplatin treatment, we examined whether cisplatin treatment alters the subcellular localization of GR. We observed that GR is translocated from the cytosol to the nucleus after cisplatin exposure in KB-3-1 and A2780 cells by immunofluorescence staining and cell fractionation (Fig. 3a, b). This observation was confirmed in vivo in xenograft mice. Administration of cisplatin resulted in nuclear translocation of GR in xenograft tumors (Fig. 3c). Furthermore, the translocation of GR was found in paired primary HNSCC patient tumors. HNSCC patients who did not respond to platinum-based therapy and had disease recurrence within 2 years after therapy were considered "cisplatin-resistant." HNSCC patients who responded to platinum-based therapy by showing no evidence of disease for 2 years after therapy were termed "cisplatin-sensitive." All patients who received platinum therapy were co-medicated with dexamethasone during treatment. In the case of cisplatin-resistant patients, GR was located mainly in the cytosol in tumors collected from patients before cisplatin or carboplatin therapy, whereas GR was predominantly stained in the nucleus in tumors collected from patients after the therapy. In contrast, the nuclear translocation of GR was not observed in cisplatin-sensitive patient group (Fig. 3d-f). Furthermore, nuclear localization of GR positively correlated with MAST1 expression in HNSCC patient tumors $(r$ $=0.823$ ) (Fig. 3g). These data suggest that cisplatin treatment activates GR by translocating it to the nucleus, which may trigger MAST1 expression and acquired platinum resistance in cancer patients.
We next explored the molecular mechanism by which cisplatin treatment translocates GR from the cytosol to the nucleus for activation. Through a series of biomolecular interaction analyses including surface plasmon resonance (SPR), differential scanning fluorimetry (DSF), and cellular thermal shift assay (CTSA), we demonstrated that there is an interaction between cisplatin and GR (Fig. 4a-c). Moreover, the binding affinity of cisplatin to GR was comparable to that of GR agonist dexamethasone (Fig. 4c). To further investigate the interaction between cisplatin and GR, we mutated cysteine residues in the ligand-binding domain (LBD) of GR (Fig. 4d). SPR kinetics analysis using cysteine mutant variants of GR-LBD revealed that WT, C643A, or C736A bound to cisplatin with similar binding strength, whereas $\mathrm{C} 622 \mathrm{~A}$ mutation abolished the binding ability, suggesting that the C622 residue in GR is responsible for binding to cisplatin (Fig. 4e). Coimmunoprecipitation revealed that cisplatin exposure results in disruption of the GR-hsp90 complex, whereas the dissociation was not observed when GR was mutated at C622 and unable to bind cisplatin (Supplementary Fig. 3). We next investigated the subcellular location of GR WT and C622A in response to cisplatin or dexamethasone treatment by immunofluorescence staining and cell fractionation. While WT GR was translocated from the cytosol to the nucleus upon cisplatin treatment, translocation of C622A GR was not observed. On the contrary, dexamethasone-induced nuclear translocation of GR regardless of C622 mutation (Fig. 4f, g). Treatment with either cisplatin or dexamethasone alone induced MAST1 promoter activity and the combination of these two drugs resulted in additive enhancement of MAST1 induction. However, mutation at C622 abolished the MAST1 promoter activation and expression induced by cisplatin but had no effect on MAST1 induction mediated by GR agonists, including dexamethasone, betamethasone, prednisolone, and triamcinolone (Fig. 4h, i; Supplementary Fig. 4a-c). These data suggest that GR undergoes dissociation from hsp90 and nuclear translocation following cisplatin binding at C622 that consequently induces MAST1 transcription through GRE in the MAST1 promoter region. Although both cisplatin and GR agonists activate GR via interaction and nuclear translocation, the interaction of cisplatin is independent of the interaction and nuclear translocation of GR mediated by the GR agonists.

GR provides cisplatin resistance to cancer cells through MAST1. We previously reported that MAST1 confers cisplatin resistance by replacing $\mathrm{cRaf}$ and reactivating MEK signaling ${ }^{33}$. To explore the role of GR in cisplatin resistance and MAPK pathway activation, we target downregulated GR using lentiviral shRNA vector or a GR antagonist mifepristone in KB-3-1 and A2780 cells. While genetic and pharmacological inhibition of GR did not alter cancer cell proliferation and MEK-ERK activation, GR 
a

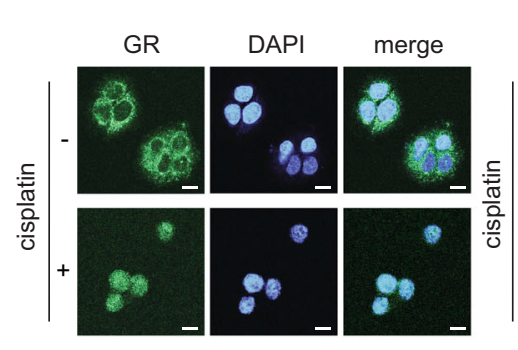

KB-3-1

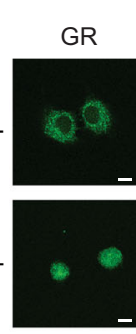

GR

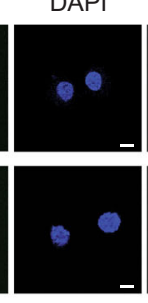

A2780

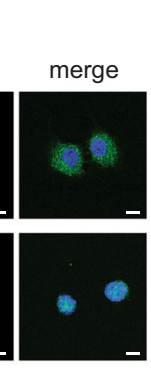

-

C

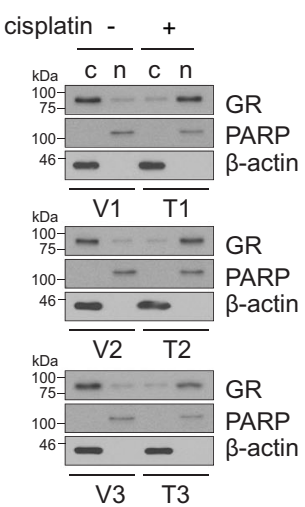

KB-3-1 xenograft tumors

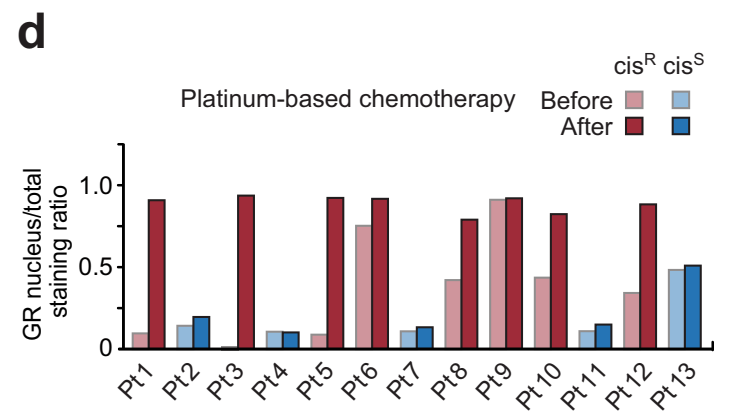

Paired platinum therapy received HNSCC patient tumors b

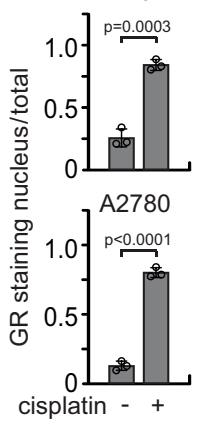

e

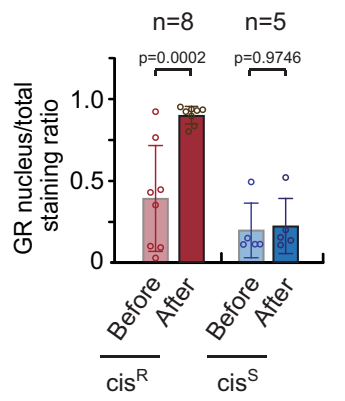

f

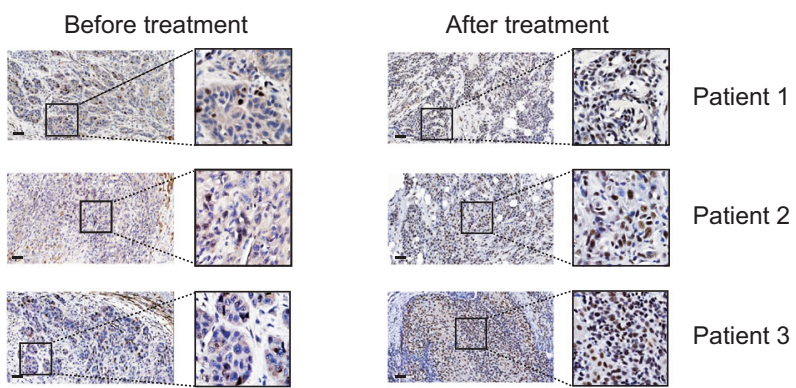

Paired platinum therapy received HNSCC patient tumors GR IHC staining g

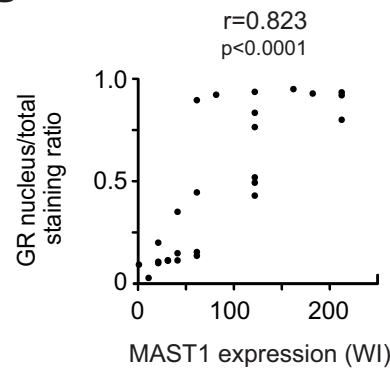

Fig. 3 Cisplatin induces GR nuclear translocation. a Immunofluorescence assay of GR in KB-3-1 and A2780 cells before and after cisplatin treatment. Nuclei were DAPI stained. $\mathbf{b}$ Western blots show the cytosolic and nuclear localization of GR upon cisplatin treatment in KB-3-1 and A2780 cells. PARP and $\beta$-actin were used as control markers for nucleus and cytosol, respectively. c: cytosol, n: nucleus. c GR localization in KB-3-1 xenograft tumors collected from cisplatin-treated (T1-T3) and vehicle-treated (V1-V3) mice. $\mathbf{d}$ and e Nuclear staining of GR in paired HNSCC patient tumor tissues collected before and after platinum treatment. The patients were either "Resistant $\left(\operatorname{cis}^{R}\right)$ " or "Sensitive $\left(\mathrm{cis}^{S}\right)$ " to platinum-based therapy. Images were quantified with ImageJ software. f Representative GR staining images of paired HNSCC patient tumors are shown. $\mathbf{g}$ The correlation between MAST1 expression and GR nuclear staining in paired tumor tissues of HNSCC patients receiving platinum therapy. Scale bars represent $10 \mu \mathrm{m}$ for $\mathbf{a}$ and $50 \mu \mathrm{m}$ for $\mathbf{f}$. Data are mean \pm SD from three random selected areas for $\mathbf{a}$, from one and three biological replicates for $\mathbf{b}$ and $\mathbf{c}$, and $n=8$ (cisplatin-resistant) and $n=5$ (cisplatinsensitive) patients for $\mathbf{e}$. Three representative tumors from 13 cases are shown for each group (before/after platinum therapy) for $\mathbf{f}$. Statistical analyses were performed by unpaired two-tailed $t$-test for $\mathbf{e}$ and two-tailed Pearson's correlation coefficient for $\mathbf{g}$. Source data are provided as a Source Data file.

inhibition significantly decreased cell viability and MEK-ERK activity when the cells were treated with a sublethal dose of cisplatin (Fig. 5a, b; Supplementary Fig. 5a, b). We then assessed the impact of enforced GR expression or activation on cisplatin resistance and MEK-ERK signaling in KB-3-1 and A2780 cells. Enforced GR expression or activation by retroviral vector bearing flag-GR or GR agonists significantly enhanced the cisplatinresistant cell survival potential and MAPK signaling activity
(Fig. 5c, d; Supplementary Fig. 5c-e). The effect of GR rescue expression on restoring cisplatin resistance was abolished when GR harbored C622A, the cisplatin-binding-deficient mutant that lacks the ability to induce MAST1 expression (Fig. 5e, f). To further explore the cisplatin resistance mechanism mediated by GR C622, we assessed cell viability, cisplatin uptake, cisplatininduced DNA damage, and MEK-ERK activity in cancer cells bearing GR WT or C622A mutant treated with increasing 
a

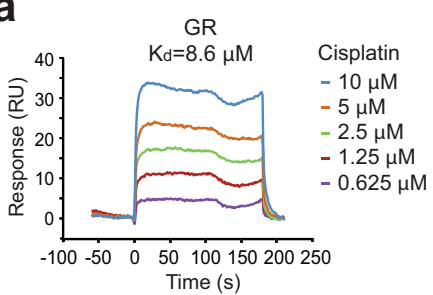

b

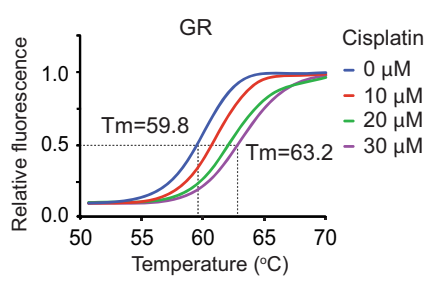

C

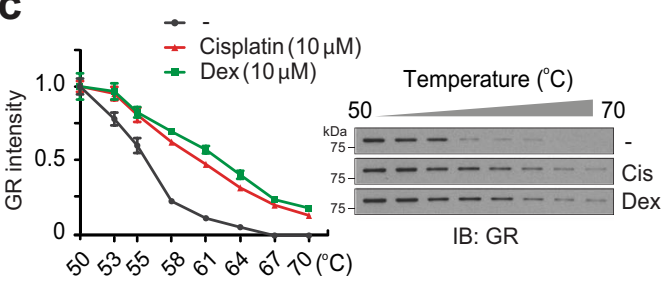

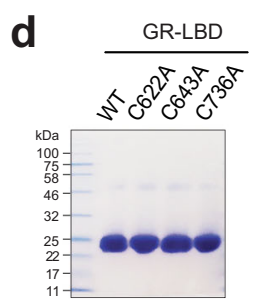
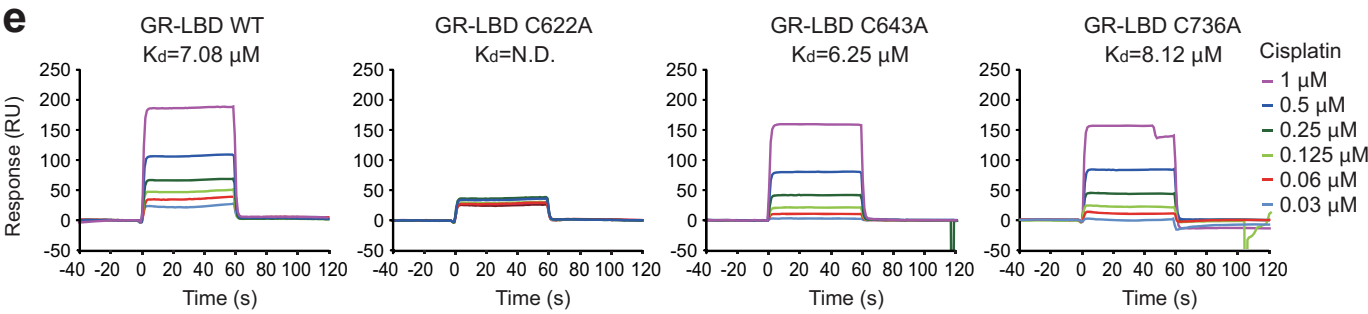

f

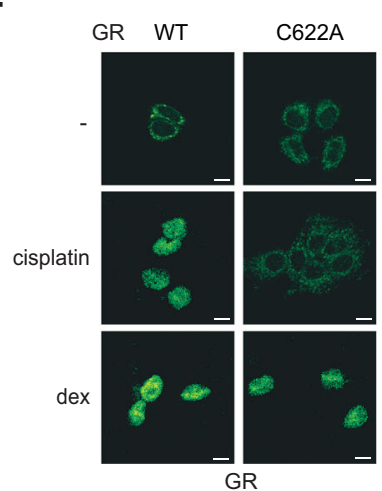

WT

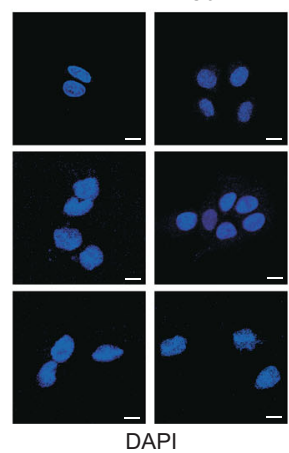

g
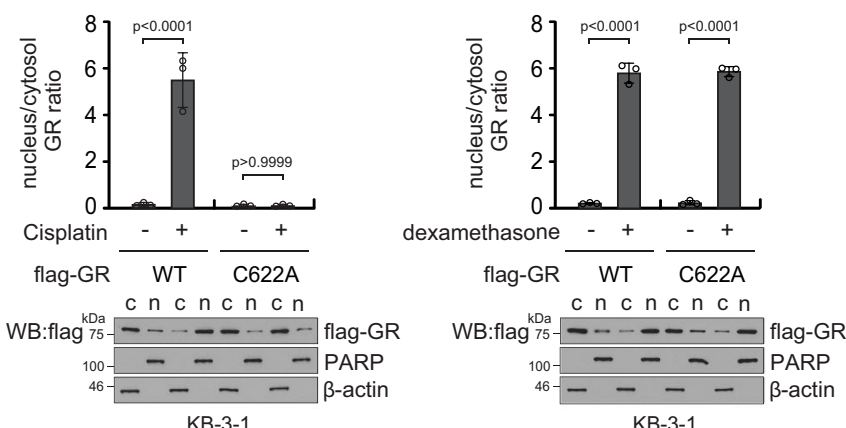

i

h

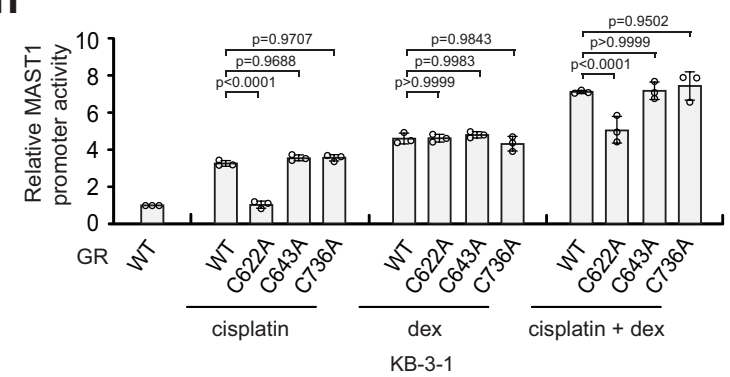

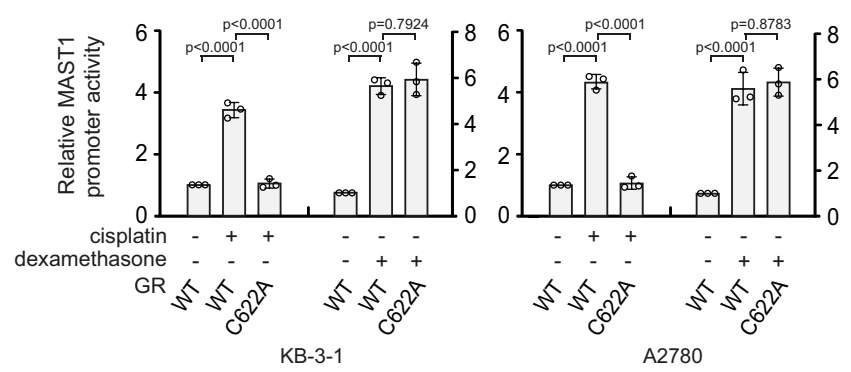

Fig. 4 Cisplatin binds to $\mathbf{C 6 2 2}$ in GR that mediates nuclear translocation and transcriptional activation. a Interaction between GR and cisplatin was determined by surface plasmon resonance (SPR) analysis and shown as a dissociation constant value. GR protein was enriched from 239T cells carrying pLHCX-flag-GR. b Differential scanning fluorimetry of GR incubated with increasing concentrations of cisplatin. c Cellular thermal shift assay using KB-3-1 cells treated with vehicle, cisplatin, or dexamethasone. $\mathbf{d}$ Coomassie-stained SDS-PAGE gel of purified recombinant GR ligand-binding domain variants, GRLBDm WT, C622A, C643A, and C736A. e Interaction between GR-LBDm variants and cisplatin is determined by SPR analyses. $\mathbf{f}, \mathbf{g}$ Effect of cisplatin and dexamethasone on GR nuclear translocation in KB-3-1 expressing GR WT or C622A mutant. KB-3-1 cells were treated with $1 \mu \mathrm{g} / \mathrm{ml}$ cisplatin or $500 \mathrm{nM}$ dexamethasone for $24 \mathrm{~h}$ followed by immunofluorescence assay (f) or nuclear/cytosol fractionation ( $\mathbf{g}$ ). $\beta$-actin and PARP were used as cytoplasmic and nuclear markers, respectively. c: cytosol, n: nucleus. Scale bars represent $10 \mu \mathrm{m}$. $\mathbf{h}$, i Effect of cisplatin and dexamethasone on MAST1 promoter activity in KB-3-1 expressing GR WT or CA mutants (h) or in KB-3-1 and A2780 cells expressing GR WT or C622A mutant (i). Compounds were administered as described in $\mathbf{f}$. Data are from one biological experiment for $\mathbf{a}$, and mean \pm SD from three independent biological experiments for the others. Statistical analyses were performed by one-way ANOVA for $\mathbf{g}-\mathbf{i}$. Source data are provided as a Source Data file.

concentrations of cisplatin. Mutation at C622 of GR attenuated cisplatin-resistant cell survival and MEK-ERK activity. However, cisplatin uptake or cisplatin-induced DNA damage was not altered in cells bearing C622A GR compared to cells with WT GR (Fig. 6a-c). These data suggest that GR may confer cisplatin resistance through interaction with cisplatin that mediates MAPK pathway activation.

To further investigate whether GR confers cisplatin resistance through MAST1, we tested whether ectopic overexpression of MAST1 can reverse the attenuated cell viability and MAPK 
a

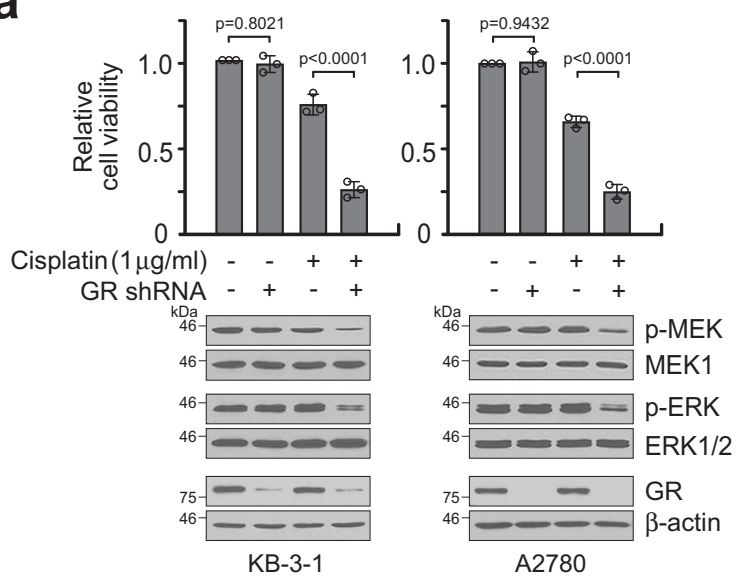

C

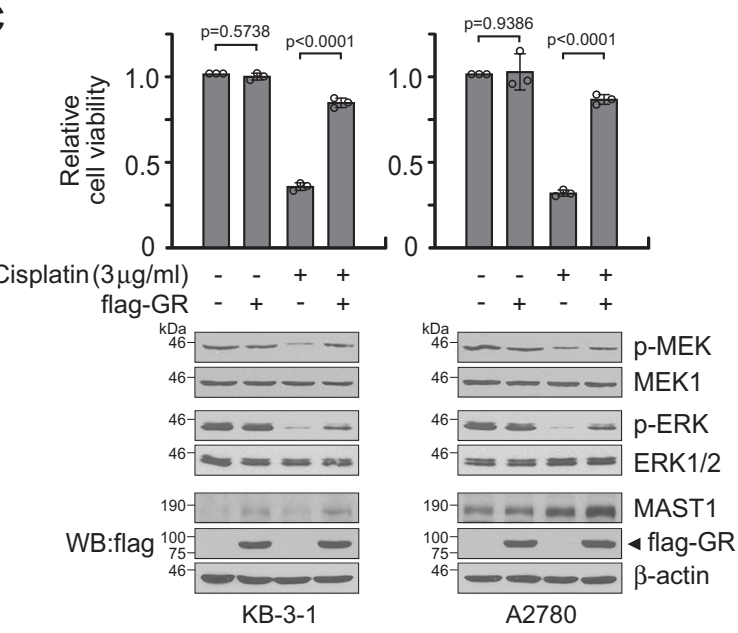

e

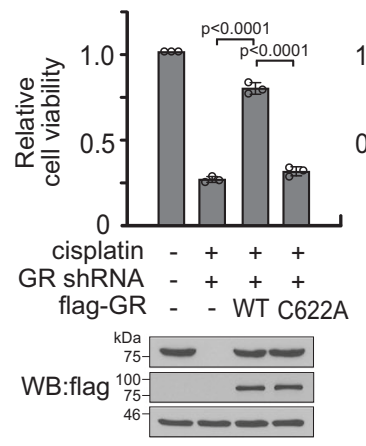

KB-3-1

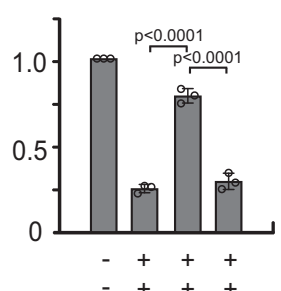

- - WT C622A

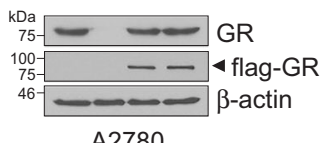

b

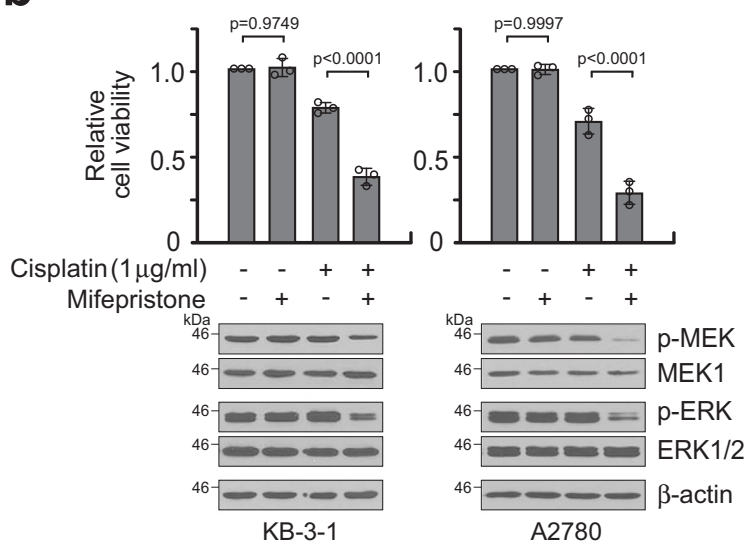

d

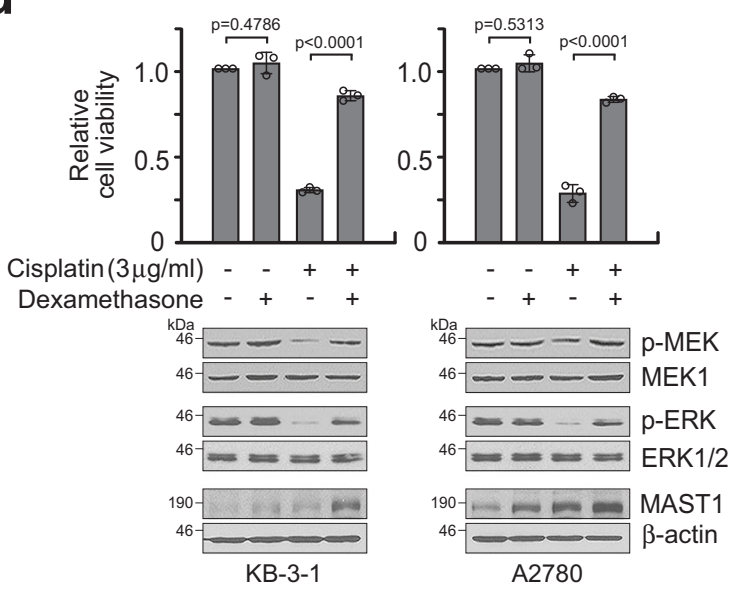

f

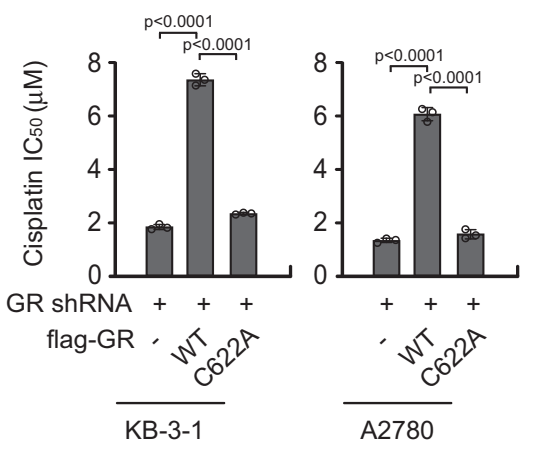

Fig. 5 GR C622 contributes to cisplatin resistance through MAST1. a, b Cell viability and MEK/ERK activity in GR target downregulated KB-3-1 and A2780 cells by GR shRNA (a) or mifepristone $(10 \mu \mathrm{M})(\mathbf{b})$ in the presence and absence of cisplatin. MEK and ERK activity was assessed by S221 MEK and T202/Y204 ERK phosphorylation. c, d Cell viability and MEK/ERK in GR enhanced KB-3-1 and A2780 cells by GR overexpression (c) or dexamethasone $(500 \mathrm{nM})(\mathbf{d})$ in the presence and absence of cisplatin. e Cell viability of KB-3-1 and A2780 cells carrying GR WT or C622A in response to cisplatin. $\mathbf{f}$ Cisplatin IC 50 of KB-3-1 and A2780 cells harboring GR WT or C622A. Data are mean \pm SD from three independent biological experiments. Error bars represent SD and statistical analyses were performed by one-way ANOVA. Source data are provided as a Source Data file.

activity in GR target downregulated cells. Stable overexpression of active MAST1 rescued the decreased cisplatin-resistant cell proliferation and MEK-ERK phosphorylation in cells with GR shRNA or mifepristone (Fig. 7a, b; Supplementary Fig. 6a, b). Similar results were obtained in vivo when MAST1 was overexpressed in GR knockdown tumors in xenograft mice (Fig. 7c-e; Supplementary Fig. 6c). On the contrary, knockdown of MAST1 abolished cisplatin-resistant cancer cell proliferation, tumor growth, and MEK-ERK phosphorylation obtained by enforced GR expression or activation in vitro (Fig. 7f, g; Supplementary Fig. 6d, e) and in vivo (Fig. 7h-j; Supplementary Fig. $6 \mathrm{f}$. In all, $2 \mathrm{mg} / \mathrm{kg}$ and $4 \mathrm{mg} / \mathrm{kg}$ of cisplatin was administered to adequately study the effect of GR-MAST1 modulation on cisplatin sensitization and resistance, respectively. These data 
a
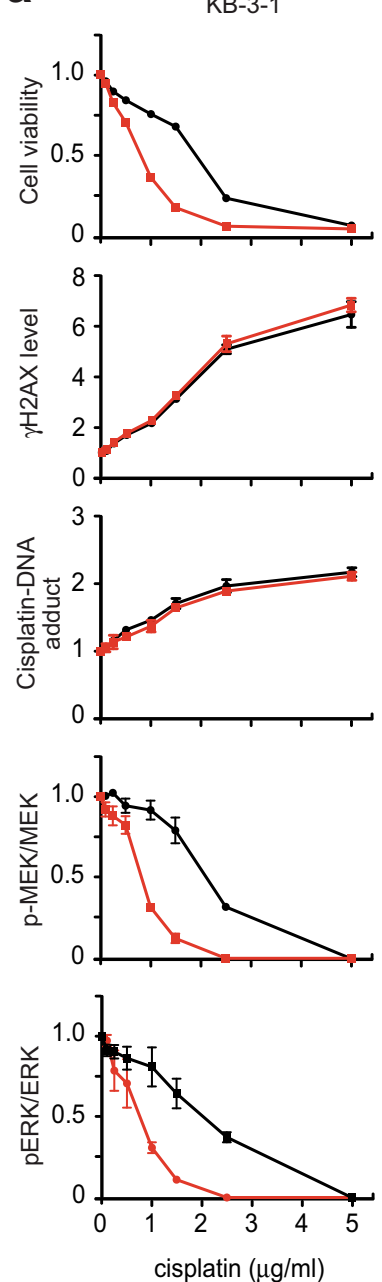

A2780
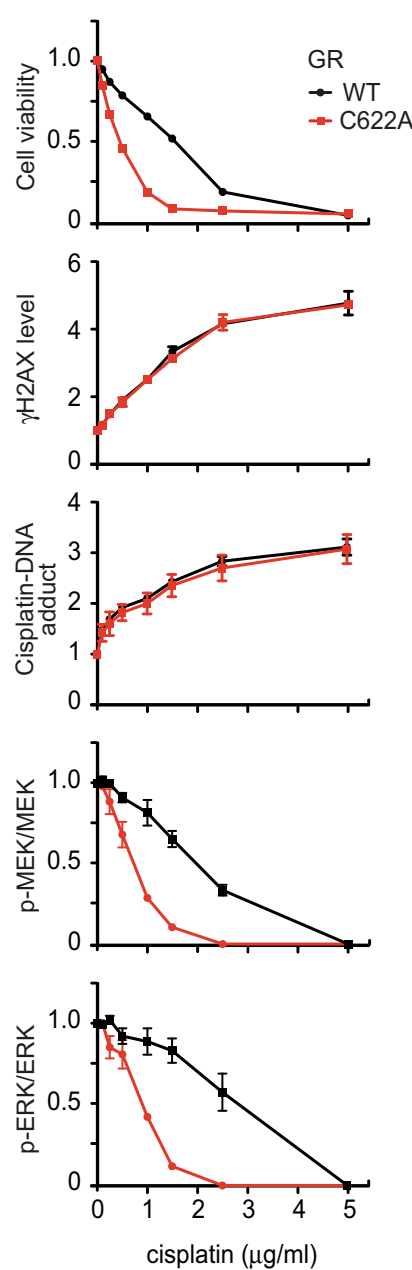

b
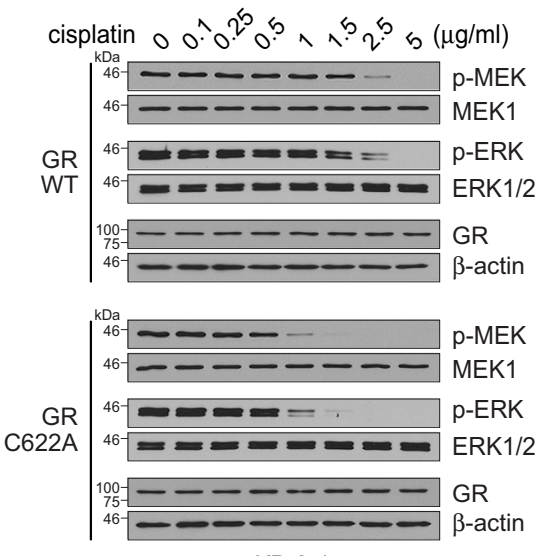

KB-3-1

C
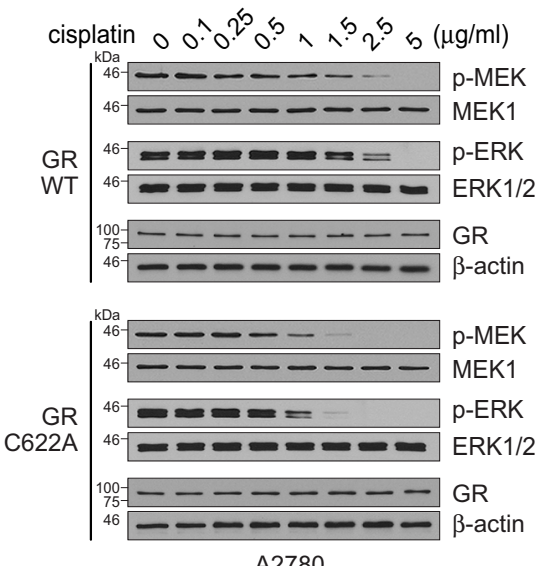

Fig. 6 GR C622 is crucial for cisplatin resistance through MEK-ERK activation but not DNA damage response. a Dose-dependent analyses of cell viability, $\gamma \mathrm{H} 2 \mathrm{AX}$, cisplatin-DNA adduct, MEK and ERK phosphorylation in KB-3-1 and A2780 cells carrying GR WT or C622A upon cisplatin treatment. b, c Representative Western blot of MEK-ERK pathway change in KB-3-1 (b) and A2780 (c) cells carrying GR WT or C622A upon treatment with increasing concentrations of cisplatin. Data are from three independent experiments and mean \pm SEM for blot analyses and mean \pm SD for others. Source data are provided as a Source Data file.

collectively suggest that GR confers cisplatin resistance by inducing the gene expression of MAST1.

Lestaurtinib abolishes cisplatin and dexamethasone-induced cisplatin resistance. Our findings indicate that GR agonists that are often given with platinum-based chemotherapy, promote acquired cisplatin resistance by enhancing MAST1 expression. Thus, we examined the effect of targeting MAST1 with a small molecule inhibitor lestaurtinib on cisplatin re-sensitization in cancer cell lines and patient-derived tumor organoids treated with GR agonists. Dexamethasone, betamethasone, prednisolone, or triamcinolone restored cisplatin-resistant cancer cell survival and MEK-ERK phosphorylation, whereas treatment with the MAST1 inhibitor diminished the elevated MEK-ERK activity and cisplatin resistance in GR agonist-treated tumor cells (Fig. 8a, b; Supplementary Figs. $7 \mathrm{a}-\mathrm{c}$ and $8 \mathrm{a})$. We next evaluated the effect of lestaurtinib on dexamethasone-induced cisplatin-resistant tumor growth in vivo in patient-derived xenograft (PDX) mouse models of head and neck cancer and ovarian cancer. Administration of dexamethasone abolished the effect of cisplatin on tumor growth and proliferation, whereas treatment with lestaurtinib in the dexamethasone-treated group fully revived cisplatin sensitivity and even further attenuated tumor growth compared to the group treated with cisplatin alone in both PDX models bearing tumors derived from head and neck cancer or ovarian cancer patients (Fig. 8c, d; Supplementary Fig. 8b, c). Known alternative lestaurtinib targets including Trk and FLT3 were not expressed in tumor cells we studied (Supplementary Fig. 9a). In addition, the inhibitory effect of lestaurtinib on dexamethasone-induced cisplatin-resistant cell growth was abolished in cells are lacking MAST1 but not JAK2. (Supplementary Fig. 9b-d). These data suggest that lestaurtinib provides cisplatin sensitivity by inhibiting MAST1. Lastly, to address the effects of lestaurtinib on the anti-inflammatory activity of dexamethasone during chemotherapy, we established the ID8-luc syngeneic mouse model. C57BL/6 mice treated with dexamethasone and cisplatin displayed greater tumor growth than mice that received vehicle or cisplatin. In agreement with observations in PDX models, lestaurtinib significantly abolished tumor growth potential in dexamethasonetreated mice (Fig. 8e). Quantification of 12 representative proand anti-inflammatory mouse cytokines in plasma revealed that dexamethasone effectively decreased pro-inflammatory cytokines, including IL-6, IL-23, IL-1 $\alpha$, TNF- $\alpha$, MCP-1, GM-CSF, IFN- $\beta$ and elevated secretion of anti-inflammatory cytokines IL-27 and 
a

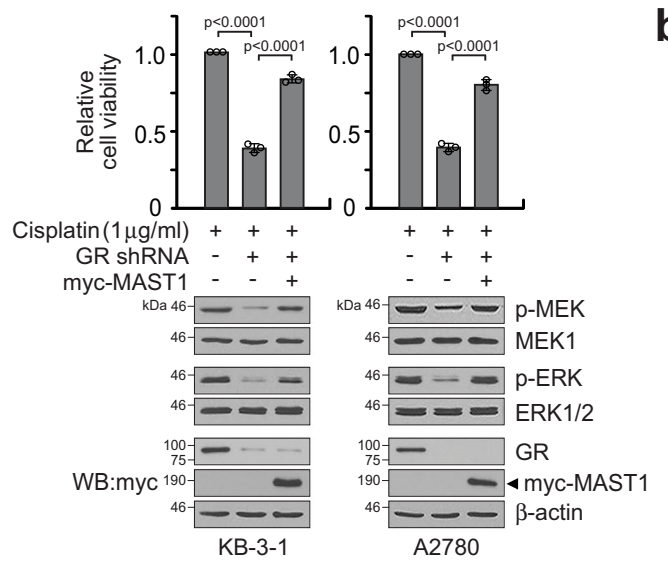

C

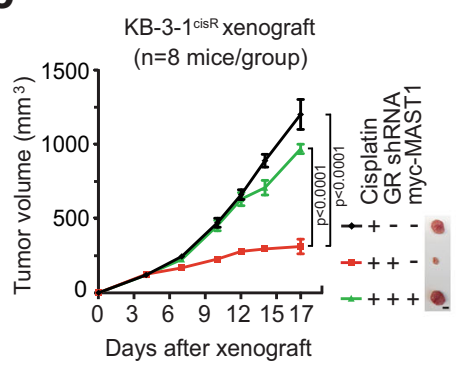

d

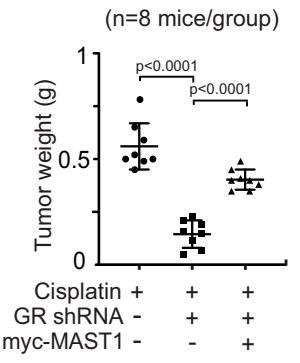

b

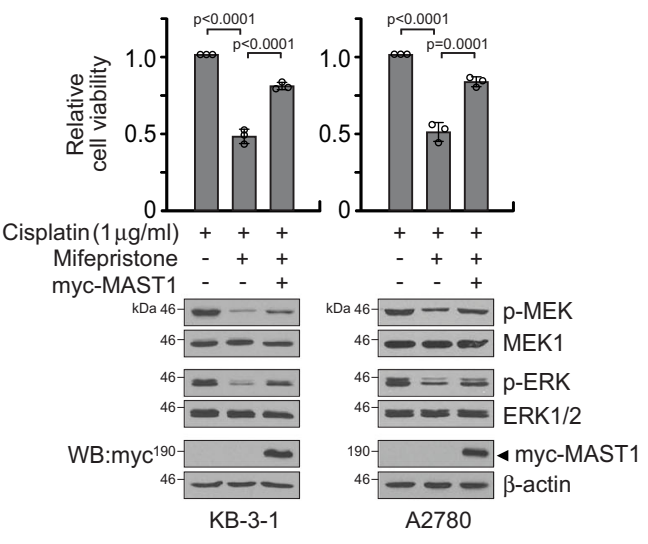

$\mathbf{f}$

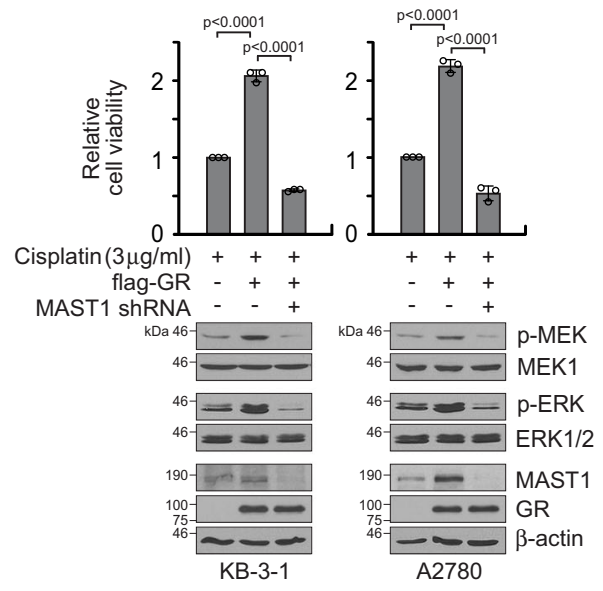

9

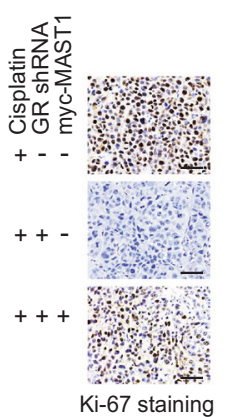

e

Cisplatin +++ GR ShRNA - + + myc-MAST1 - -+
kDa $46-\infty$

$\mathrm{kDa} 46-\mathrm{P}-\mathrm{MEK}$
$\mathrm{4}=\mathrm{MEK} 1$

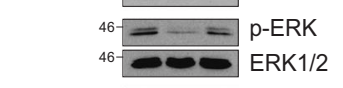

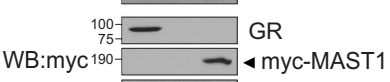
- $-\infty$-actin KB-3-1 ${ }^{\text {cisR }}$ xenograft tumor lysates
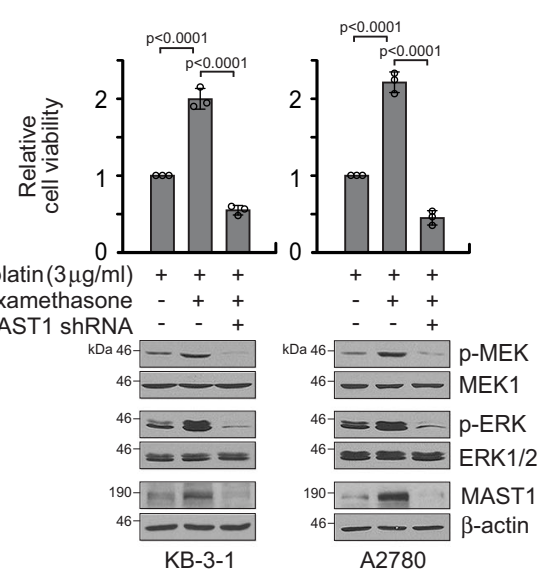

h

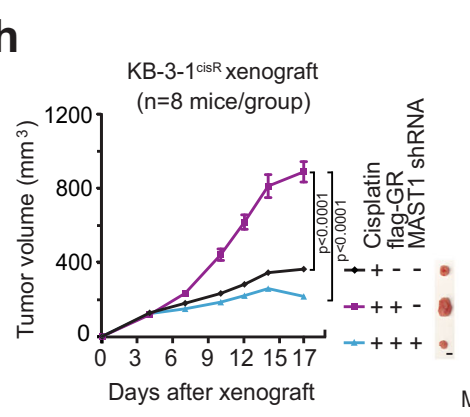

i

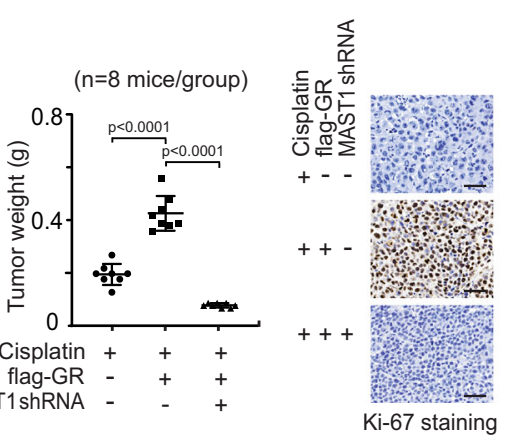

j

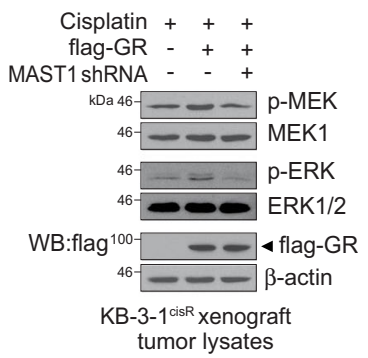

IL-10. However, lestaurtinib did not influence cytokine release or modulation mediated by dexamethasone (Fig. 8f). These data collectively show that lestaurtinib effectively attenuates cisplatinresistant tumor growth that is induced by dexamethasone without affecting the anti-inflammatory activity of dexamethasone in vivo.

\section{Discussion}

While platinum-based chemotherapy is the standard front-line therapy for patients with solid malignant tumors, treatment is often accompanied by toxic side effects and thus glucocorticoids are widely used as co-medication to reduce inflammation and relieve symptoms. Despite the conventional usage of GR agonists 
Fig. 7 GR promotes cisplatin-resistant cell survival and tumor growth via MAST1-MEK-ERK. a, b Effect of MAST1 overexpression on cell viability and MEK/ERK activity in GR attenuated KB-3-1 and A2780 cells with cisplatin treatment. GR was target downregulated by GR shRNA (a) or mifepristone (10 $\mu \mathrm{M})(\mathbf{b})$. MEK and ERK activity was assessed by S221 MEK and T202/Y204 ERK phosphorylation. c-e Effect of MAST1 overexpression on tumor growth of xenograft mice bearing GR knockdown KB-3-1 cells. Mice were treated with cisplatin ( $2 \mathrm{mg} / \mathrm{kg}$ i.p. twice/week) from 4 days after xenograft. Tumor volume (c), tumor weight and Ki-67 staining (d), and phosphorylation of MEK and ERK in tumors (e) are shown. $\mathbf{f}, \mathbf{g}$ Effect of MAST1 knockdown on cell viability and MEK/ERK activity in GR enhanced KB-3-1 and A2780 cells with cisplatin treatment. GR was enhanced by GR overexpression (f) or dexamethasone $(500 \mathrm{nM})(\mathbf{g}) \cdot \mathbf{h}-\mathbf{j}$ Effect of MAST1 knockdown on tumor growth of xenograft mice bearing GR overexpressed KB-3-1 cells. Mice were treated with cisplatin ( $4 \mathrm{mg} / \mathrm{kg}$ i.p. twice/week) from 4 days after xenograft. Tumor volume (h), tumor weight and Ki-67 IHC staining (i), and MEK and ERK activity in tumors (j) are shown. Scale bars represent $5 \mathrm{~mm}$ for $(\mathbf{c}, \mathbf{h})$, and $50 \mu \mathrm{m}$ for $(\mathbf{d}, \mathbf{i})$. Data are from three independent biological experiments for $(\mathbf{a}, \mathbf{b}, \mathbf{f}, \mathbf{g})$ and $n=8$ mice/group for $\mathbf{c}-\mathbf{e}$ and $\mathbf{h}-\mathbf{j}$. One representative data are shown for (right panels of $\mathbf{d}$ and $\mathbf{i} ; \mathbf{e}$ and $\mathbf{j}$ ). Data are mean \pm SD for $(\mathbf{a}, \mathbf{b}, \mathbf{d}, \mathbf{f}, \mathbf{g}, \mathbf{i})$ and mean $\pm \mathrm{SEM}$ for $\mathbf{c}$ and $\mathbf{h}$. Statistical analyses were performed by two-way ANOVA for $\mathbf{c}$ and $\mathbf{h}$, and one-way ANOVA for others. Source data are provided as a Source Data file.

as antiemetic regimens for chemotherapy, how GR signaling cross-talks with platinum resistance in human cancer has not been fully characterized. Here, we delineate a unique molecular mechanism by which GR is activated by cisplatin and steroid, and how the activation of GR contributes to platinum resistance in human cancers through MAST1 using a series of preclinical studies and analysis of platinum-treated patient tumor specimens. We found that GR is activated upon cisplatin treatment by cisplatin binding to GR and transporting GR from the cytosol to the nucleus. Further activation of GR occurs by its agonists such as dexamethasone in a cisplatin-independent manner. The activated GR contributes to cisplatin resistance by serving as a transcription factor of MAST1, which consequently reactivates MEK. We also clinically validated our findings by demonstrating a correlation between the nuclear localization of GR and MAST1 expression levels as well as cisplatin resistance in primary tumor tissue samples collected from cancer patients before and after treatment with platinum-containing regimens.

We demonstrated that cisplatin induces GR activation by binding to GR at C622, which leads to MAST1 expression and phosphorylation of MEK and ERK. Cisplatin mediates GR nuclear translocation and consequently promotes GR activity as a transcription factor. We previously demonstrated that cisplatin also binds to MEK1 at C142 that mediates disruption of the MEK1-cRaf complex allowing MAST1 to serve as a substitute for cRaf in MAPK signaling ${ }^{33}$. During these processes, the tethering of cisplatin to GR or MEK did not alter its uptake or its ability to induce DNA damage or repair, suggesting that cisplatin has a dual effect in cancer cells. In addition to its conventional role in DNA crosslinking, cisplatin may serve as a signaling mediator in cancer cells to either translocate or dissociate protein-protein interactions that are critical in post-target mechanisms of resistance. In support, other studies report interactions between cisplatin and cellular factors 38,39 .

Our study revealed that cisplatin binds to GR at cysteine 622 . Mutation at C622 abolished the interaction between cisplatin and GR and retained GR in the cytosol, which impaired the induction of MAST1 transcription and cisplatin resistance. However, C622A mutation did not alter the GR agonist-mediated nuclear translocation and transcriptional activity of GR. Therefore, cisplatin and GR agonists such as dexamethasone may not share the same GR interaction and activation mechanism in cells. Dexamethasone is a larger moiety that binds to a canonical deep pocket in $\mathrm{GR}^{40}$, whereas, cisplatin is a smaller molecule that attaches to the cysteine residue, and these may lead to different binding states. GR forms a multiprotein complex in cells with hsp90 and others ${ }^{7-9}$. It is possible that cisplatin integrated into GR alters the shape of the complex or stabilizes a state that hsp90 cannot bind. We found that MAST1 is protected from proteasomal degradation by binding to hsp $90^{34}$. However, hsp90 $\beta$ but not hsp90a induced MAST1 stabilization whereas both equivalently have chaperone activities on GR, suggesting a differential involvement of hsp90 isoforms in MAST1 stability and GR activation process ${ }^{34,41}$. Future studies are warranted to define the structural basis by which cisplatin and steroids coordinate GR activation.

We demonstrated that MAST1 expression and stability are enhanced by GR and hsp90 $\beta$, respectively, upon cisplatin exposure. Levels of MAST1 increased by GR and maintained by hsp $90 \beta$-mediated protection against degradation may lead to a consequent surge in activated MAST1. It is plausible that MAST1 is further activated or inhibited via its phospho-dynamics through cellular kinases or phosphatases in cancer cells. For instance, potential MAST1 interacting partners from the STRING database including membrane-associated guanylate kinase MAGI-2 or serine/threonine-protein phosphatase PPP2R2C/B could be alternative MAST1 regulators that drive cancer cisplatin resistance.

Ectopic expression of MAST1 fully restored cisplatin resistance lost by GR attenuation. In line with this result, genetic or pharmacological enhancement of GR did not offer cisplatin resistance to cancer cells when MAST1 was absent. These studies indicate that although GR transcriptionally regulates a variety of genes, GR mainly programs cisplatin-resistant pro-survival signaling in human cancers through transcriptional regulation of MAST1.

GR signaling is engaged in a variety of functions in malignant cells. In lymphocytes, GR is known to arrest cell growth and induce apoptosis, thus glucocorticoids are considered effective anti-cancer agents for lymphatic cancers ${ }^{42}$. However, GR is known to play a contrasting role in solid malignant tumors. For instance, a high level of GR was linked with differential enhancement of epithelial-mesenchymal transition and cell adhesion in breast cancer ${ }^{43}$. A recent study demonstrated that GR increased tumor heterogeneity and metastasis through ROR1 in breast cancer ${ }^{44}$. Moreover, activation of GR caused increased proliferation and invasion in metastatic colon cancer through $\mathrm{CDK}^{45}$. In our study, we provide clinical evidence that GR nuclear translocation positively correlates with MAST1 expression and platinum resistance in head and neck cancer patients. This suggests that the GR-MAST1 signaling axis could be a promising predictive marker for acquired platinum resistance in human cancer. In addition, cisplatin but not other chemotherapeutic agents such as mitomycin $\mathrm{C}$ or camptothecin induced GR activation in cancer cells, suggesting that the signaling axis may serve as an effective biomarker specifically for patients receiving platinum-based therapy.

Lastly, pharmacological studies using PDX and syngeneic mouse models indicate that co-treatment with GR agonist dexamethasone and cisplatin fosters tumor growth. This suggests that the frequent use of steroids as a component of supportive oncology, namely as a major element of the cisplatin antiemetic regimen, may need to be re-examined. Targeting MAST1 using a 
a

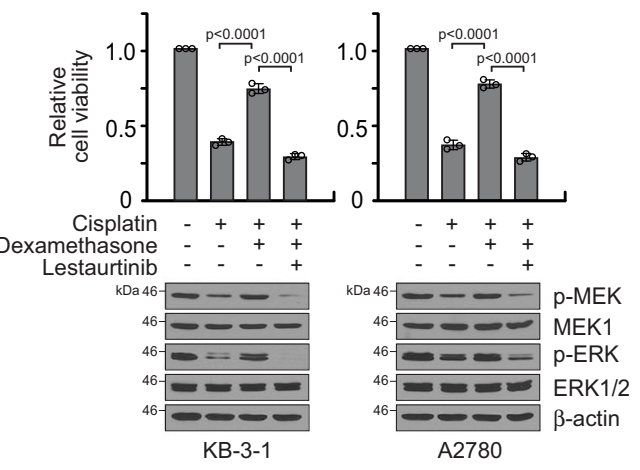

C

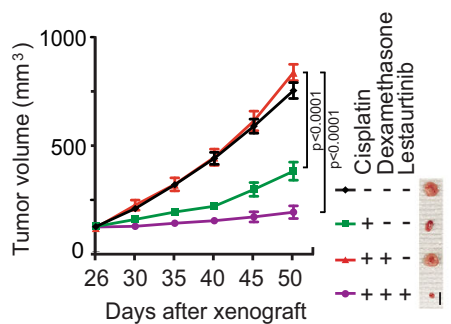

Head and neck cancer PDX ( $n=8$ mice/group)

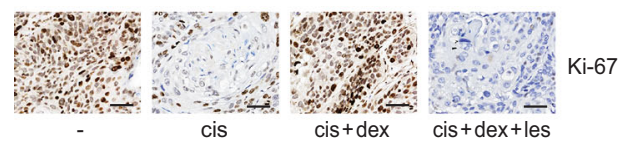

b
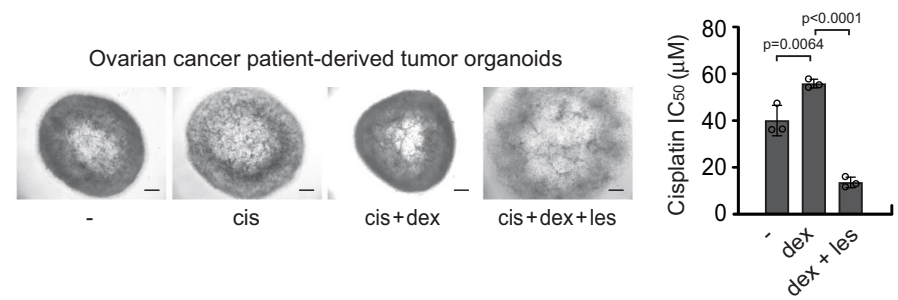

d

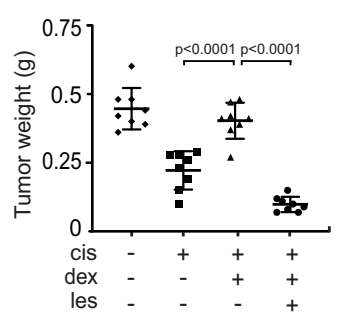

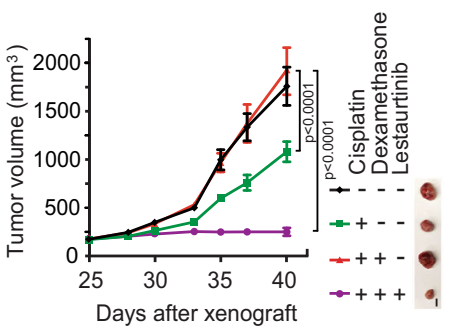

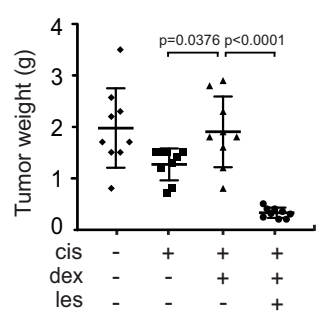

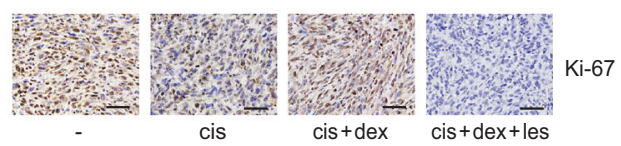

e

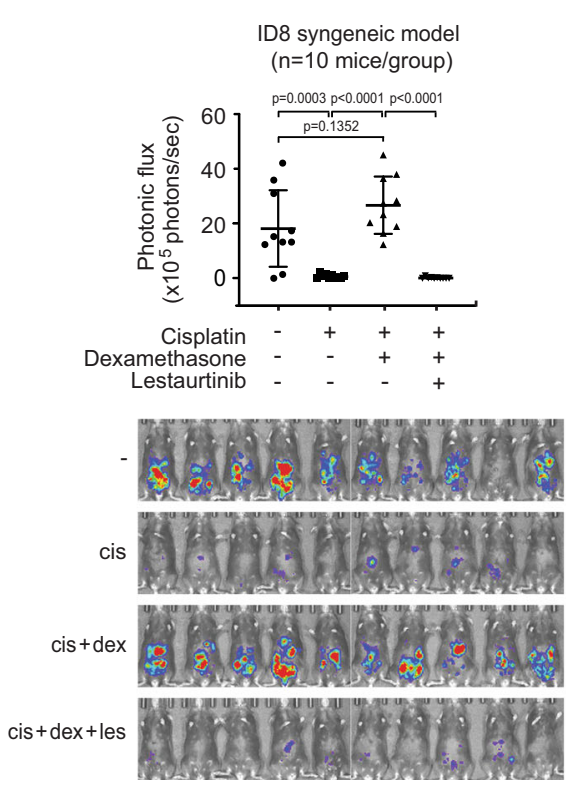

f
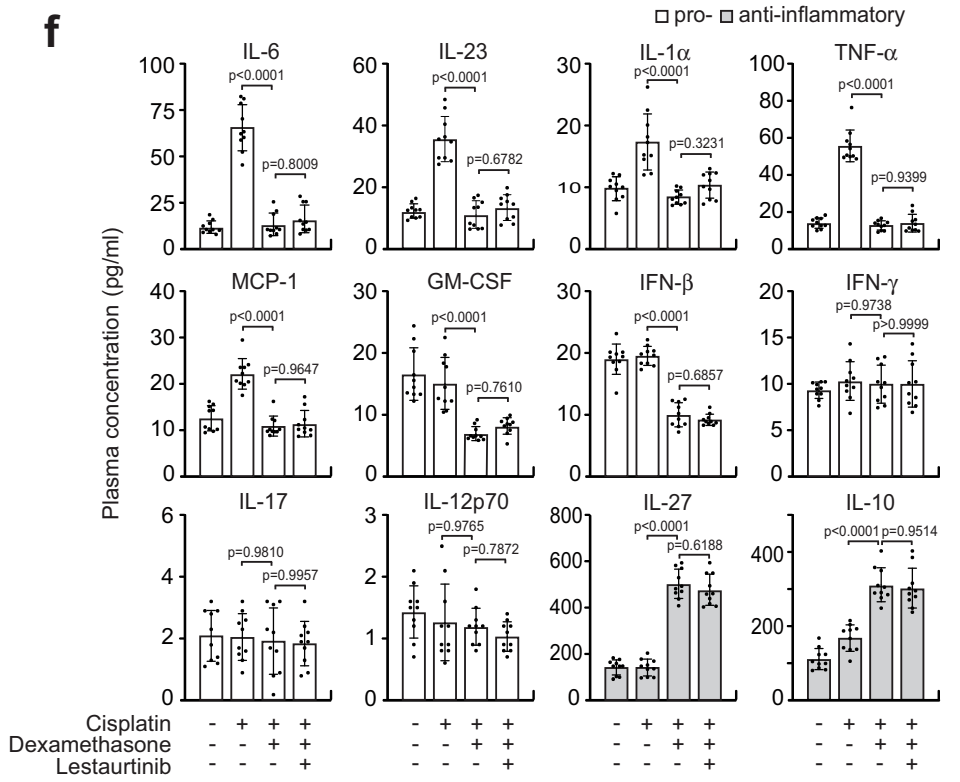

$-+++$

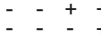

$-+++$

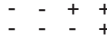

ID8 syngeneic model

Fig. 8 Lestaurtinib suppresses dexamethasone-mediated GR-MAST1 activation and cisplatin resistance while not affecting the anti-inflammatory property of dexamethasone. a Effect of lestaurtinib on cell viability and MAPK activity of dexamethasone and cisplatin co-treated KB-3-1 and A2780 cells. Cells were treated with $100 \mathrm{nM}$ lestaurtinib, $500 \mathrm{nM}$ dexamethasone, and $3 \mu \mathrm{g} / \mathrm{ml}$ cisplatin. b Effect of lestaurtinib on growth of ovarian cancer patientderived xenograft tumor organoid treated with cisplatin and dexamethasone. Images (left) and cisplatin $\mathrm{IC}_{50}$ (right) of organoids are shown. Single cell suspended organoids were used for $\mathrm{IC}_{50}$ analysis. Scale bars represent $100 \mu \mathrm{m}$. c, d Effect of lestaurtinib on tumor growth of HNSCC (c) and ovarian cancer (d) PDX mice receiving cisplatin and dexamethasone treatment. Cisplatin ( $5 \mathrm{mg} / \mathrm{kg}$ i.p. twice/week), dexamethasone $(0.1 \mathrm{mg} / \mathrm{kg}$ i.p. twice/week) and lestaurtinib (20 mg/kg s.c. daily) were treated. Tumor volume (left), tumor weight (right), and Ki-67 IHC staining of PDX tumors (bottom) are shown for HNSCC and ovarian PDX models. Scale bars represent $5 \mathrm{~mm}$ for tumor images and $50 \mu \mathrm{m}$ for Ki-67 staining. e, $\mathbf{f}$ Effect of lestaurtinib on tumor growth and inflammation of ID8-luc syngeneic mice receiving cisplatin and dexamethasone treatment. Syngeneic mice were treated as PDX mice. Average photonic flux and BLI (e) and plasma inflammation related cytokine profile (f) from 10 mice/group at week 8 are shown. Data are mean \pm SD from three independent biological replicates for $\mathbf{a}$ and $\mathbf{b}$. In vivo data are from 8 mice for $\mathbf{c}, 9$ mice for $\mathbf{d}, 10$ mice for $\mathbf{e}$ and $\mathbf{f}$. Data are mean \pm SEM for tumor volume (left panels in $\mathbf{c}$ and $\mathbf{d}$ ) and SD for tumor weight (right panels in $\mathbf{c}$ and $\mathbf{d}$ ), photonic flux (e), and cytokine profiling (f). Statistical analyses were performed by two-way ANOVA for tumor volume and one-way ANOVA for the rest. Source data are provided as a Source Data file. 
well-tolerated small molecule kinase inhibitor lestaurtinib may be a method to abrogate the possible effects of steroids on tumor growth as treatment with lestaurtinib effectively re-sensitized tumors to cisplatin treatment while not interrupting the antiinflammatory activity. Although further optimization of treatment conditions in the clinic is warranted, our study provides a mechanism by which GR agonists along with platinum drugs induce resistance and demonstrate that use of a MAST1 inhibitor may provide more effective platinum-based chemotherapy regimens for patients receiving a GR agonist such as dexamethasone as an anti-inflammatory drug.

\section{Methods}

Reagents. Human lentiviral short hairpin RNA (shRNA) clones targeting GR, PR, NFAT, ATF2, CAR, CBF, JAK2, and MAST1 were obtained from Open Biosystems. Human GR, GR-LBD, PR, and MAST1 were flag or myc tagged by PCR and subcloned into pLHCX-derived or pET53-DEST Gateway destination vectors as previously described ${ }^{46}$. MAST1 promoter reporter was obtained from Switchgear Genomics. MAST1 GRE mutant promoter reporter and GR-LBDm (GR-LBDF602S/A605V/V702A/E705G/M752 $\mathrm{T}^{47}$ ) and its cysteine mutant variants were generated using site-directed mutagenesis reagents from Agilent. Primer sequences are listed in Supplementary Table 1. CellTiter-Glo Luminescent Viability assay and LightSwitch Luciferase Assay were obtained from Promega and Active Motif, respectively. TF Activation Profiling Array was purchased from Signosis. The LEGENDplex Mouse Inflammation Panel was obtained from Biolegend. Cisplatin, dexamethasone, betamethasone, prednisolone, triamcinolone, and mifepristone were purchased from Sigma-Aldrich. Mitomycin and camptothecin were from Selleckchem. Lestaurtinib was obtained from Tocris Bioscience. A2780 and ID8 cells were purchased from Sigma-Aldrich. KB-3-1 cells were obtained as previously described ${ }^{48}$. KB-3-1 is a derivative of HeLa commonly used in multiple drug resistance studies ${ }^{49}$. 293T cells were from American Type Culture Collection. All cell lines were authenticated by STR profiling. Ovarian PDX tumor for organoid culture was purchased from Jackson Laboratory.

Antibodies. Antibodies against GR (12041/D6H2L), PR (3153/C89F7), phosphoMEK1/2 (S221) (2338/166F8), MEK1/2 (9126/47E6), phospho-ERK1/2 (T202/ Y204) (4376/20G11), ERK1/2 (4695/137F5), myc (2276/9B11), hsp90 (4877/ C45G5), phospho-histone H2A.X (S139) (9718/20E3), PARP (9532/46D11), panTrk (92991/A7H6R), and Jak2 (3230/D2E12) were obtained from Cell Signaling Technology. MAST1 antibodies (NBP2-17228 and NBP1-81453) were obtained from Novus Biologicals. Antibodies against FLAG (F7425 and F1804/M2) and $\beta$ actin (A1978/AC-15) were purchased from Sigma-Aldrich. Antibodies against cisplatin-modified DNA (ab103261/CP9/19) and Ki-67 (ab92742/EPR3610) were obtained from Abcam. Anti-ATF-2 antibody (sc-242/F2BR-1) and anti-FLT3 (sc479/C-20) antibody were from Santa Cruz Biotechnology. Antibodies against CEBPZ (31-163) and NFATC1 (MA3-024/7A6) were purchased from ProSci and Invitrogen, respectively. Anti-CAR/NR1I3 antibody (PP-N4111-00/N4111) was obtained from R\&D Systems.

Cell and organoid culture. A2780 cells were cultured in RPMI 1640 medium with $10 \%$ FBS. KB-3-1, 293T, and ID8 cells were cultured in Dulbecco Modified Eagle Medium (DMEM) with 10\% FBS. Lentivirus production, infection, and stable cell selection for gene knockdown or overexpression and protein overexpression were previously described ${ }^{46,50}$. Cells were treated for $48 \mathrm{~h}$ with $1 \mu \mathrm{g} / \mathrm{ml}$ and $3 \mu \mathrm{g} / \mathrm{ml}$ cisplatin to study the effect of GR-MAST1 modulation on cisplatin sensitization and resistance, respectively, unless specified. For organoid culture, ovarian PDX tumors were minced into $1 \mathrm{~mm}$ diameter pieces and crushed. The homogenates were digested with $2.5 \mathrm{mg} / \mathrm{ml}$ Type II collagenase in basal culture media [advanced DMEM/F12 medium supplemented with 1x GlutaMAX, 1\% HEPES, and 1\% penicillin-streptomycin] for $25 \mathrm{~min}$ at $37^{\circ} \mathrm{C}$. The cell pellets were washed twice with red blood cell lysis buffer and resuspended in $75 \%$ Matrigel at a concentration of $1 \times 10^{6} / \mathrm{ml}$. Approximately $1.5 \times 10^{4}$ cells were loaded into each well of a 48 -well plate and cultured in general culture medium [advanced DMEM/F12 medium supplemented with $100 \mathrm{ng} / \mathrm{ml}$ R-spondin $1,100 \mathrm{ng} / \mathrm{ml} \mathrm{Noggin,} 50 \mathrm{ng} / \mathrm{ml}$, EGF, 10 $\mathrm{ng} / \mathrm{ml}$ FGF-10, $10 \mathrm{ng} / \mathrm{ml} \mathrm{FGF} 2,2 \% \mathrm{~B}-27,10 \mathrm{mmol} / \mathrm{l}$ nicotinamide, $1.25 \mathrm{mmol} / \mathrm{l} \mathrm{N}$ acetylcysteine, $1 \mu \mathrm{mol} / \mathrm{l}$ prostaglandin $\mathrm{E} 2,10 \mu \mathrm{mol} / \mathrm{l} \mathrm{SB202190,} 500 \mathrm{nmol} / \mathrm{l} \mathrm{A83-01}$, and $10 \mu \mathrm{mol} / \mathrm{l} \mathrm{Y-27632}]^{51,52}$. Organoids were single cell suspended and cell viability was measured using CellTiter-Glo Luminescent Viability Assay ${ }^{33}$.

Transcriptome profiling and quantitative RT-PCR. RNA was isolated using RNeasy Mini Kit (Qiagen) from tumors collected from KB-3-1 xenograft mice treated or non-treated with cisplatin. Poly-A RNA-sequencing was conducted by LC Sciences according to the manufacturer's instruction. Quantitative RT-PCR was conducted with High-Capacity cDNA Reverse Transcription Kit (Applied Biosystems), Universal SYBR Green Supermix (Bio-Rad), and primers designed for MAST1 using PrimerBank.
Cell viability assay and cisplatin sensitivity analysis. Cells were seeded on 96 well plates one day prior to the addition of compounds with indicated concentrations for $48 \mathrm{~h}$. Cell viability was measured using CellTiter-Glo Luminescent Viability Assay. Half-maximal inhibitory concentration $\left(\mathrm{IC}_{50}\right)$ values were obtained using GraphPad Prism 8 to measure cisplatin sensitivity.

Transcription factor activation profiling. KB-3-1 cells were treated with $5 \mu \mathrm{g} / \mathrm{ml}$ cisplatin for $24 \mathrm{~h}$ and nuclear proteins were extracted. The activities of 96 essential transcription factors were monitored by TF Activation Profiling Plate Array II (Signosis) according to the manufacturer's protocol ${ }^{53,54}$.

ChIP and promoter reporter assay. ChIP assays were performed using chromatin immunoprecipitation assay (Millipore). In brief, endogenous GR or PR was immunoprecipitated from KB-3-1 cells. The MAST1 promoter region in the DNA eluted from immune-precipitates was amplified by PCR. For MAST1 promoter reporter assay, MAST1 promoter variants were transfected into KB-3-1 or A2780 cells under indicated conditions and the promoter reporter activity was measured using LightSwitch dual luciferase assay system (SwitchGear Genomics).

Surface plasmon resonance (SPR). To study cisplatin and GR interaction, $1 \mu \mathrm{M}$ of recombinant human GR-LBD WT or CA mutants were coupled to CM5 sensor chip. Various concentrations of cisplatin were prepared in 0.01 M HEPES pH 7.4, $0.005 \% \mathrm{v} / \mathrm{v}$ Surfactant $\mathrm{P} 20$, and $0.15 \mathrm{M} \mathrm{NaCl}$ solution, and injected over GR-LBD at $30 \mu \mathrm{l} / \mathrm{min}$ speed for $3 \mathrm{~m}$ at $20^{\circ} \mathrm{C}$. Multiple-cycle kinetic analysis was performed to quantify interaction between cisplatin and GR-LBD variants. The raw sensorgrams were blank-subtracted and dissociation constant $\left(K_{\mathrm{d}}\right)$ values were obtained using BIA Evaluation Software v2.1 (GE Healthcare).

Differential scanning fluorimetry and cellular thermal shift assay. Differential scanning fluorimetry (DSF) was performed by incubating $10 \mu \mathrm{M}$ of recombinant GR with $0,10,20$, or $30 \mu \mathrm{M}$ of cisplatin for $10 \mathrm{~m}$ at $20^{\circ} \mathrm{C}$. GR-cisplatin mixture was employed to thermal shift reactions. Fluorescence intensity was recorded by a realtime PCR system and the data were analyzed using Protein Thermal Shift ${ }^{\mathrm{TM}}$ Software v1.3. For cellular thermal shift assay, KB-3-1 cells were treated with DMSO, cisplatin or dexamethasone for $24 \mathrm{~h}$. Collected cells were applied to 3 freeze-thaw cycles in PBS. A series of cell aliquots were heated at 50, 53, 55, 58, 61 64,67 , and $70^{\circ} \mathrm{C}$ for $3 \mathrm{~min}$. The GR in the cell lysates of each aliquot was quantified by western blot analysis ${ }^{33,55}$.

Immunofluorescence microscopy. KB-3-1 cells seeded on glass coverslips were immersed in PHEMO buffer (68 mM PIPES, $25 \mathrm{mM}$ HEPES, $15 \mathrm{mM}$ EGTA, and 3 $\mathrm{mM} \mathrm{MgCl} 2$ ) with $0.05 \%$ glutaraldehyde, $3.7 \%$ formaldehyde, and $0.5 \%$ Triton X100 , and blocked in PBS containing 5\% goat serum ${ }^{46}$. Cells were then incubated with GR antibody in PBS containing 2.5\% goat serum followed by incubation with Alexa Fluor 488 conjugated goat anti-rabbit IgG. Samples were washed and mounted with antifade mounting medium with DAPI. Images were taken using Leica SP8 confocal microscope.

Immunohistochemistry staining. Paraffin-embedded paired tumor specimens collected from head and neck cancer patients before and after receiving platinumbased chemotherapy were obtained from the Head and Neck Satellite Tissue Bank of Emory University. The study in human specimens was approved by the Institutional Review Board at Emory University. The study was conducted in compliance with ethical standards and good clinical practice. Clinical samples were collected with informed consent of the individuals or their guardians under Health Insurance Portability and Accountability Act (HIPAA) guidelines. IHC staining was conducted as previously described ${ }^{33,55}$. In brief, GR and MAST1 staining was performed by incubating the sections with GR antibody (1:400 dilution) and MAST1 antibody (1:200). Tumors collected from KB-3-1 or patient-derived xenograft (PDX) mice were stained with Ki-67 antibody (1:1000). The final immunoreactive score was determined by weighted index (WI) multiplying the intensity $(0-3+)$ and extent of positivity scores (\%) of stained cells ${ }^{56}$.

In vivo studies and cytokine profiling. Animal studies were performed according to protocols reviewed and approved by the Institutional Animal Care and Use Committee of Emory University. Nude mice (Hsd:Athymic Nude-Foxn $1^{\text {nu }}$, female, 6-week-old, Envigo) were injected with $0.5 \times 10^{6} \mathrm{~KB}-3-1$ cells. For in vivo model of cisplatin-resistant cancer, nude mice bearing KB-3-1 xenografts were administered with a sub-effective dose $(0.5 \mathrm{mg} / \mathrm{kg})$ and a higher dose $(5 \mathrm{mg} / \mathrm{kg})$ on day 5 and 10 , respectively. For GR functional studies, cisplatin $(2 \mathrm{or} 4 \mathrm{mg} / \mathrm{kg})$ were administered two times a week by intraperitoneal (i.p.) injection when tumor sizes reached up to $100 \mathrm{~mm}^{3}$. For PDX studies, head and neck cancer patient tumor was implanted into NOD scid gamma mice (female, 6-week-old, Jackson Laboratory). Approximately $1500 \mathrm{~mm}^{3}$ sized tumors were excised, evenly diced, and implanted in the flank of nude mice. Ovarian cancer PDX model was obtained from Jacksons Laboratory. The mice were randomly divided into groups when the tumor size reached $100 \mathrm{~mm}^{3}$. For syngeneic mouse model, luciferase gene was transduced into murine ovarian ID8 cells and injected into C57BL/6 mice (female, 6-week-old, 
Envigo) by intraperitoneal (i.p.) injection. Cisplatin $(5 \mathrm{mg} / \mathrm{kg})$ and dexamethasone $(0.1 \mathrm{mg} / \mathrm{kg})$ were given by i.p. injection twice a week. Lestaurtinib $(20 \mathrm{mg} / \mathrm{kg} / \mathrm{day})$ was administered by subcutaneous injection. KB-3-1 or PDX tumors were measured and volumes were calculated as $4 \pi / 3 \times(\text { width/2) })^{2}$ (length/2). Blinding and allocation concealment were used. For syngeneic ID8 mouse model, tumor growth was monitored by bioluminescence imaging (BLI) analysis as described ${ }^{57}$. Blood was harvested at the experimental endpoint and mouse inflammatory cytokines, including IL-6, IL-23, IL-1 $\alpha$, TNF- $\alpha$, MCP-1, GM-CSF, IFN- $\beta$, IFN- $\gamma$, IL-17, IL12 p70, IL-27, IL-10, and IL-1 $\beta$ in plasma were quantified by flow cytometry analysis. IL-1 $\beta$ was not detected in the C57BL/6 mice plasma.

Statistical analysis. Statistical analyses were performed using GraphPad Prism 9.0. Sample size was not predetermined by statistics. One representative experiment of multiple experiments is shown for each immunoblotting figure panel. Error bars represent mean \pm standard deviation (SD), except tumor growth curves and blot density analysis in Fig. 6a, which denote mean \pm standard error of the mean (SEM). Statistical significance was based on two-tailed Student's $t$-test for Figs. 1a bottom, $1 \mathrm{~d}, 1 \mathrm{f}, 2 \mathrm{~h}$, and $3 \mathrm{e}$, Pearson's correlation coefficient for $3 \mathrm{~g}$, and one-way or two-way ANOVA for all other data of multiple-comparisons. Statistical analyses are based on assumptions of normal distribution and homogeneity of variances. $P$-values of $<$ 0.05 were regarded as statistically significant.

Reporting summary. Further information on research design is available in the Nature Research Reporting Summary linked to this article.

\section{Data availability}

The RNA-seq datasets generated in the course of this study are available on the NCBI GEO database under the accession number GSE179263 Publicly available datasets obtained from http://firebrowse.org/?cohort=OV and https://string-db.org/ were used in the study. Source data are provided with this paper.

Received: 15 October 2020; Accepted: 6 July 2021; Published online: 16 August 2021

\section{References}

1. Dasari, S. \& Tchounwou, P. B. Cisplatin in cancer therapy: molecular mechanisms of action. Eur. J. Pharmacol. 740, 364-378 (2014).

2. Franzoi, M. A. et al. Clinical outcomes of platinum-based chemotherapy in patients with advanced breast cancer: an 11-year single institutional experience. Breast 57, 86-94 (2021).

3. Leal, F. \& Garcia-Perdomo, H. A. Effectiveness of platinum-based chemotherapy in patients with metastatic prostate cancer: systematic review and meta-analysis. Clin. Genitourin. Cancer 17, e627-e644 (2019).

4. Aapro, M. S. \& Alberts, D. S. Dexamethasone as an antiemetic in patients treated with cisplatin. N. Engl. J. Med. 305, 520 (1981).

5. Rich, W. M., Abdulhayoglu, G. \& DiSaia, P. J. Methylprednisolone as an antiemetic during cancer chemotherapy-a pilot study. Gynecol. Oncol. 9, 193-198 (1980).

6. Lu, N. Z. et al. International Union of Pharmacology. LXV. The pharmacology and classification of the nuclear receptor superfamily: glucocorticoid, mineralocorticoid, progesterone, and androgen receptors. Pharm. Rev. 58, 782-797 (2006).

7. Pratt, W. B. \& Toft, D. O. Regulation of signaling protein function and trafficking by the hsp90/hsp70-based chaperone machinery. Exp. Biol. Med. (Maywood) 228, 111-133 (2003).

8. Pratt, W. B. \& Toft, D. O. Steroid receptor interactions with heat shock protein and immunophilin chaperones. Endocr. Rev. 18, 306-360 (1997).

9. Kirschke, E., Goswami, D., Southworth, D., Griffin, P. R. \& Agard, D. A. Glucocorticoid receptor function regulated by coordinated action of the Hsp90 and Hsp70 chaperone cycles. Cell 157, 1685-1697 (2014).

10. Giguere, V., Hollenberg, S. M., Rosenfeld, M. G. \& Evans, R. M. Functional domains of the human glucocorticoid receptor. Cell 46, 645-652 (1986).

11. Wang, B. et al. Ahil regulates the nuclear translocation of glucocorticoid receptor to modulate stress response. Transl. Psychiatry 11, 188 (2021).

12. Mazaira, G. I., Echeverria, P. C. \& Galigniana, M. D. Nucleocytoplasmic shuttling of the glucocorticoid receptor is influenced by tetratricopeptide repeat-containing proteins. J. Cell Sci. 133, jcs238873 (2020).

13. Sun, K. et al. SIRT2 suppresses expression of inflammatory factors via Hsp90glucocorticoid receptor signalling. J. Cell Mol. Med. 24, 7439-7450 (2020).

14. Oakley, R. H. \& Cidlowski, J. A. The biology of the glucocorticoid receptor: new signaling mechanisms in health and disease. J. Allergy Clin. Immunol. 132, 1033-1044 (2013).

15. Rhen, T. \& Cidlowski, J. A. Antiinflammatory action of glucocorticoids-new mechanisms for old drugs. N. Engl. J. Med. 353, 1711-1723 (2005).
16. Muzikar, K. A., Nickols, N. G. \& Dervan, P. B. Repression of DNA-binding dependent glucocorticoid receptor-mediated gene expression. Proc. Natl Acad. Sci. USA 106, 16598-16603 (2009).

17. Shimba, A. \& Ikuta, K. Control of immunity by glucocorticoids in health and disease. Semin. Immunopathol. 42, 669-680 (2020).

18. Hesketh, P. J. \& Sanz-Altamira, P. Aprepitant, dexamethasone, and palonosetron in the prevention of doxorubicin/cyclophosphamide-induced nausea and vomiting. Support Care Cancer 20, 653-656 (2012).

19. Shih, A. \& Jackson, K. C. 2nd Role of corticosteroids in palliative care. J. Pain. Palliat. Care Pharmacother. 21, 69-76 (2007).

20. Ge, H. et al. Dexamethasone reduces sensitivity to cisplatin by blunting p53dependent cellular senescence in non-small cell lung cancer. PLOS ONE 7, e51821 (2012).

21. Pang, D., Kocherginsky, M., Krausz, T., Kim, S. Y. \& Conzen, S. D. Dexamethasone decreases xenograft response to Paclitaxel through inhibition of tumor cell apoptosis. Cancer Biol. Ther. 5, 933-940 (2006).

22. Chen, Y. X. et al. Dexamethasone enhances cell resistance to chemotherapy by increasing adhesion to extracellular matrix in human ovarian cancer cells. Endocr.-Relat. Cancer 17, 39-50 (2010).

23. Wagenblast, J. et al. Does dexamethasone inhibit the antineoplastic effect of cisplatin and docetaxel in head and neck cancer cells? Anticancer Res. 30, 123-127 (2010)

24. Lu, Y. S. et al. Glucocorticoids enhance cytotoxicity of cisplatin via suppression of NF-\{kappa\}B activation in the glucocorticoid receptor-rich human cervical carcinoma cell line SiHa. J. Endocrinol. 188, 311-319 (2006)

25. Sorrentino, G. et al. Glucocorticoid receptor signalling activates YAP in breast cancer. Nat. Commun. 8, 14073 (2017).

26. He, L. et al. Glucocorticoid receptor signaling activates TEAD4 to promote breast cancer progression. Cancer Res. 79, 4399-4411 (2019).

27. Metzger, R. et al. ERCC1 mRNA levels complement thymidylate synthase mRNA levels in predicting response and survival for gastric cancer patients receiving combination cisplatin and fluorouracil chemotherapy. J. Clin. Oncol.: Off. J. Am. Soc. Clin. Oncol. 16, 309-316 (1998).

28. Jun, H. J. et al. ERCC1 expression as a predictive marker of squamous cell carcinoma of the head and neck treated with cisplatin-based concurrent chemoradiation. Br. J. Cancer 99, 167-172 (2008).

29. Olaussen, K. A. A new step ahead for the consideration of ERCC1 as a candidate biomarker to select NSCLC patients for the treatment of cetuximab in combination with cisplatin. Cancer Biol. Ther. 8, 1922-1923 (2009).

30. Nakayama, K. et al. Prognostic value of the Cu-transporting ATPase in ovarian carcinoma patients receiving cisplatin-based chemotherapy. Clinical cancer research: an official journal of the American Association for. Cancer Res. 10, 2804-2811 (2004).

31. Aida, T. et al. Expression of copper-transporting P-type adenosine triphosphatase (ATP7B) as a prognostic factor in human endometrial carcinoma. Gynecologic Oncol. 97, 41-45 (2005).

32. Moreno-Smith, M. et al. ATP11B mediates platinum resistance in ovarian cancer. J. Clin. Investig. 123, 2119-2130 (2013).

33. Jin, L. et al. MAST1 drives cisplatin resistance in human cancers by rewiring cRaf-independent MEK activation. Cancer Cell 34, 315-330 e317 (2018).

34. Pan, C. et al. Hsp90B enhances MAST1-mediated cisplatin resistance by protecting MAST1 from proteosomal degradation. J Clin. Investig. 129, 4110-4123 (2019).

35. $\mathrm{Hu}, \mathrm{T}$. et al. PDK2 induces cisplatin-resistance in lung adenocarcinoma via transcriptional regulation of CNNM3. J. Drug Target 27, 460-465 (2019).

36. Wang, Q. et al. CDK20 interacts with KEAP1 to activate NRF2 and promotes radiochemoresistance in lung cancer cells. Oncogene 36, 5321-5330 (2017).

37. Kelly, E. J., Sandgren, E. P., Brinster, R. L. \& Palmiter, R. D. A pair of adjacent glucocorticoid response elements regulate expression of two mouse metallothionein genes. Proc. Natl Acad. Sci. USA 94, 10045-10050 (1997).

38. Moltgen, S. et al. Cisplatin protein binding partners and their relevance for platinum drug sensitivity. Cells $\mathbf{9}, 1322$ (2020).

39. Karasawa, T., Sibrian-Vazquez, M., Strongin, R. M. \& Steyger, P. S. Identification of cisplatin-binding proteins using agarose conjugates of platinum compounds. PLoS ONE 8, e66220 (2013).

40. Bledsoe, R. K. et al. Crystal structure of the glucocorticoid receptor ligand binding domain reveals a novel mode of receptor dimerization and coactivator recognition. Cell 110, 93-105 (2002).

41. Morishima, Y. et al. Chaperone activity and dimerization properties of Hsp90alpha and Hsp90beta in glucocorticoid receptor activation by the multiprotein Hsp90/Hsp70-dependent chaperone machinery. Mol. Pharm. 94, 984-991 (2018).

42. Shipman, G. F., Bloomfield, C. D., Smith, K. A., Peterson, B. A. \& Munck, A. The effects of glucocorticoid therapy on glucocorticoid receptors in leukemia and lymphoma. Blood 58, 1198-1202 (1981).

43. Pan, D., Kocherginsky, M. \& Conzen, S. D. Activation of the glucocorticoid receptor is associated with poor prognosis in estrogen receptor-negative breast cancer. Cancer Res. 71, 6360-6370 (2011). 
44. Obradovic, M. M. S. et al. Glucocorticoids promote breast cancer metastasis. Nature 567, 540-544 (2019).

45. Tian, D., Tian, M., Han, G. \& Li, J. L. Increased glucocorticoid receptor activity and proliferation in metastatic colon cancer. Sci. Rep. 9, 11257 (2019).

46. Jin, L. et al. Glutamate dehydrogenase 1 signals through antioxidant glutathione peroxidase 1 to regulate redox homeostasis and tumor growth. Cancer Cell 27, 257-270 (2015).

47. Seitz, T. et al. Enhancing the stability and solubility of the glucocorticoid receptor ligand-binding domain by high-throughput library screening. J. Mol. Biol. 403, 562-577 (2010).

48. Richert, N., Akiyama, S., Shen, D., Gottesman, M. M. \& Pastan, I. Multiply drug-resistant human $\mathrm{KB}$ carcinoma cells have decreased amounts of a 75$\mathrm{kDa}$ and a $72-\mathrm{kDa}$ glycoprotein. Proc. Natl Acad. Sci. USA 82, 2330-2333 (1985).

49. Hall, M. D. et al. Say no to DMSO: dimethylsulfoxide inactivates cisplatin, carboplatin, and other platinum complexes. Cancer Res. 74, 3913-3922 (2014).

50. Jin, L. et al. p90 RSK2 mediates antianoikis signals by both transcriptiondependent and -independent mechanisms. Mol. Cell. Biol. 33, 2574-2585 (2013).

51. Hill, S. J. et al. Prediction of DNA repair inhibitor response in short-term patient-derived ovarian cancer organoids. Cancer Discov. 8, 1404-1421 (2018).

52. Drost, J. et al. Organoid culture systems for prostate epithelial and cancer tissue. Nat. Protoc. 11, 347-358 (2016).

53. Toubal, A. et al. SMRT-GPS2 corepressor pathway dysregulation coincides with obesity-linked adipocyte inflammation. The. J. Clin. Investig. 123, 362-379 (2013).

54. Jin, L. et al. The PLAG1-GDH1 axis promotes anoikis resistance and tumor metastasis through CamKK2-AMPK signaling in LKB1-deficient lung cancer. Mol. Cell 69, 87-99 e87 (2018).

55. Martinez Molina, D. et al. Monitoring drug target engagement in cells and tissues using the cellular thermal shift assay. Science 341, 84-87 (2013).

56. Adam, J. et al. Renal cyst formation in Fh1-deficient mice is independent of the Hif/Phd pathway: roles for fumarate in KEAP1 succination and Nrf2 signaling. Cancer Cell 20, 524-537 (2011)

57. Alesi, G. N. et al. RSK2 signals through stathmin to promote microtubule dynamics and tumor metastasis. Oncogene 35, 5412-5421 (2016).

\section{Acknowledgements}

We appreciate Dr. Anthea Hammond for critical reading of the manuscript. This work was supported in part by NIH grants R01 CA207768 (S.K.), R01 CA175316 (S.K.), F31 CA246889 (A.C.B.), R37 CA249305 (L.J.), P50 CA217691 (S.R. and H.F), Developmental Funds from the Winship Cancer Institute of Emory University (S.K.), and the Emory Integrated Cellular Imaging Microscopy Core of the Winship Cancer Institute compre- hensive cancer center award P30 CA138292. A.C.B. is an NIH pre-doctoral fellow. S.K. is a Georgia Cancer Coalition Scholar and an American Cancer Society Basic Research Scholar.

\section{Author contributions}

G.Z.C., N.F.S., and D.M.S. provided critical reagents. T.J.B. performed structural analyses K.R.M. collected clinical samples and performed histopathological study. X.W. provided clinical information. L.J. performed transcription factor profiling and data analysis. J.L. assisted in vivo experiments. C.P., J.K., J.H., A.C.B., and L.Y. performed all other experiments. S.K. and C.P. designed the study and S.K. wrote the paper.

\section{Competing interests}

S.K. and L.J. are inventors of "Use of MAST1 inhibitor in treatment of platinum-based chemotherapy" technology. The other authors declare no competing interests.

\section{Additional information}

Supplementary information The online version contains supplementary material available at https://doi.org/10.1038/s41467-021-24845-8.

Correspondence and requests for materials should be addressed to S.K.

Peer review information Nature Communications thanks Giannino Del Sal, Karolien De Bosscher and the other anonymous reviewer(s) for their contribution to the peer review of this work. Peer reviewer reports are available.

Reprints and permission information is available at http://www.nature.com/reprints

Publisher's note Springer Nature remains neutral with regard to jurisdictional claims in published maps and institutional affiliations.

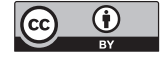

Open Access This article is licensed under a Creative Commons Attribution 4.0 International License, which permits use, sharing, adaptation, distribution and reproduction in any medium or format, as long as you give appropriate credit to the original author(s) and the source, provide a link to the Creative Commons license, and indicate if changes were made. The images or other third party material in this article are included in the article's Creative Commons license, unless indicated otherwise in a credit line to the material. If material is not included in the article's Creative Commons license and your intended use is not permitted by statutory regulation or exceeds the permitted use, you will need to obtain permission directly from the copyright holder. To view a copy of this license, visit http://creativecommons.org/ licenses/by/4.0/

(C) The Author(s) 2021 